\title{
A NOTE ON THE EQUIVALENCE AND THE BOUNDARY BEHAVIOR OF A CLASS OF SOBOLEV CAPACITIES
}

\author{
C. CHRISTOF* AND G. MÜLLER ${ }^{\dagger}$
}

\begin{abstract}
The purpose of this paper is to study different notions of Sobolev capacity commonly used in the analysis of obstacle- and Signorini-type variational inequalities. We review basic facts from nonlinear potential theory in an abstract setting that is tailored to the study of $W^{1, p_{\text {- }} \text { and }}$ $W^{1-1 / p, p}$-capacities, and we prove equivalency results that relate several approaches found in the literature to each other. Motivated by applications in contact mechanics, we especially focus on the behavior of different Sobolev capacities on and near the boundary of the domain in question. As a result, we obtain, for example, that the most common approaches to the sensitivity analysis of Signorini-type problems are exactly the same.
\end{abstract}

Key words. capacity theory, boundary, optimal control, Signorini, variational inequalities, contact mechanics, sensitivity analysis, nonlinear potential theory, classification problems

AMS subject classifications. 28C15, 31B15, 31B25, 31C15, 49K40, 74M15

1. Introduction. In the theory of Sobolev spaces, it is not appropriate to study the pointwise behavior of functions in the almost everywhere sense inherited from the underlying measure space. This is already seen in the fact that Sobolev functions admit well-defined traces on sets that are negligible in the measure theoretical sense (cf. the classical trace theorem). To analyze the fine properties of Sobolev functions properly, it is necessary to work with capacities (cf. $[1,2,3,5,8,11,12,16]$ ). These are outer measures that take the additional regularity of the involved functions into account and allow for precise identification of the sets that are negligible in the context of Sobolev spaces. Due to this increased accuracy, capacities play a crucial role in, e.g., the characterization of the exceptional sets in Egorov-type theorems and the study of sets that are defined by pointwise constraints. Examining pointwise conditions up to sets of capacity zero - so-called polar sets - leads to the notion of a property holding "quasi everywhere" (q.e.), which is finer than the concept of "almost everywhere" (a.e.) known from the theory of Lebesgue spaces.

Problems that need to be studied in a quasi everywhere sense arise, e.g., in the optimal control and the sensitivity analysis of Signorini- and obstacle-type variational inequalities. In both of these fields, it is often necessary to characterize the tangent-, normal-, and critical cones to sets of the form

$$
\left\{v \in W_{(0)}^{1, p}(\Omega): v \geq \psi \text { in } \bar{\Omega}\right\},
$$

where $\Omega \subset \mathbb{R}^{d}$ is a bounded domain and $\psi: \bar{\Omega} \rightarrow \mathbb{R} \cup\{ \pm \infty\}$ is a given function. Such a characterization is only possible if the inequality $v \geq \psi$ in (1.1) is understood in a quasi everywhere sense. As a consequence, the concept of capacity becomes indispensable. We refer to $[6,15,17,18,21,22,23]$ for details on this topic. A particular difficulty in the setting above is inherent to Signorini-type problems, where the inequality constraint $v \geq \psi$ is nontrivial, i.e., $\psi \neq-\infty$, only on subsets of the

\footnotetext{
*Faculty of Mathematics, Technische Universität Dortmund, Dortmund, Germany.

${ }^{\dagger}$ Faculty of Mathematics, Universität Bayreuth, Bayreuth, Germany.
} 
domain boundary. For these problems, the $W_{0}^{1, p}(\Omega)$-capacity, that is most commonly used in the analysis of partial differential equations (cf. $[5,11,16]$ ), is not applicable, and one has to resort to alternative notions of capacity to obtain a meaningful quasi everywhere sense for the study of (1.1).

Several different approaches can and have been taken to this end. In [17], Mignot employed the theory of Dirichlet spaces (which is only applicable in the case $p=2$ ) and the capacity of the space $W^{1,2}(\Omega)$ to obtain a meaningful capacity on the power set $\mathcal{P}(\bar{\Omega})$ of the closure $\bar{\Omega}$ (cf. [17, page 150, Exemple 2] and [15]). In [6], on the other hand, Betz worked with the $W_{0}^{1, p}$-capacity of an open set $\Omega^{\prime}$ satisfying $\bar{\Omega} \subset \Omega^{\prime}$ to define a reasonable quasi everywhere sense on the closure of the domain $\Omega$. Lastly, it is also a natural choice to define a capacity based on the space $W^{1-1 / p, p}(\partial \Omega)$ on the boundary $\partial \Omega$ - especially since, in the case of Signorini problems, the inequality constraint $v \geq \psi$ is commonly interpreted in the sense of traces (cf. [15, Example 6] and also $[6,9,13])$.

The purpose of this paper is to prove that all of the above approaches are, in fact, the same. To be more precise, in what follows, we show that the capacities associated with the spaces $W^{1, p}(\Omega), W^{1, p}\left(\mathbb{R}^{d}\right), W^{1-1 / p, p}(\partial \Omega)$, and $W_{0}^{1, p}\left(\Omega^{\prime}\right)$ (where, again, $\Omega^{\prime}$ is an open set satisfying $\bar{\Omega} \subset \Omega^{\prime}$ ) are all equivalent on the power set $\mathcal{P}(\partial \Omega)$ of the boundary $\partial \Omega$, provided $\Omega$ is a strong bounded Lipschitz domain. While some results on the equivalence of different Sobolev capacities are known (see, e.g., [16, Theorem 2.38] for a theorem on $W_{0}^{1, p}$-capacities on different domains), to the best of our knowledge, the relationship between the latter four capacities has not been studied so far. With the following analysis we close this gap. We further hope that our results can alleviate some of the confusion that arose in the field of contact mechanics due to the multitude of different approaches to boundary capacities found in the literature. The outline of this paper is as follows:

In Section 2, we recall basic facts from nonlinear potential theory that are needed for our analysis. Here, we precisely define the concepts of "capacity" and "quasi everywhere" in a general function space setting and discuss the existence and uniqueness of quasi continuous representatives in depth. We include a detailed review of the latter topics for two reasons: On the one hand, to work with the spaces $W^{1, p}(\Omega), W^{1, p}\left(\mathbb{R}^{d}\right)$, $W^{1-1 / p, p}(\partial \Omega)$, and $W_{0}^{1, p}\left(\Omega^{\prime}\right)$, we require an abstract setting that is slightly more general than the ones usually found in the literature. Since we are also interested in the case $p \neq 2$, the classical theory of Dirichlet spaces (cf. $[7,12,15,17])$ is not suitable for our needs, and since we do not exclusively work with zero boundary conditions, we cannot simply resort to the common $W_{0}^{1, p}$-theory (cf. $[5,11,16]$ ), either. As a consequence, it is necessary to discuss the results and notions of nonlinear potential theory needed in Sections 3 to 5 in greater detail. On the other hand, we include a detailed review of the known theory to keep this paper self-contained. We hope that in doing so, we can make the topic of (boundary) capacities more accessible to those readers who are interested in, e.g., contact mechanics but unfamiliar with the field of potential theory.

In Section 3, we demonstrate that the theory of Section 2 indeed enables us to study the spaces $W^{1, p}(\Omega), W^{1, p}\left(\mathbb{R}^{d}\right), W^{1-1 / p, p}(\partial \Omega)$, and $W_{0}^{1, p}\left(\Omega^{\prime}\right)$. We further use this third section to clarify notation and to discuss preliminary results on the properties of $W^{1, p_{-}}$and $W_{0}^{1, p}$-capacities. The latter include, e.g., a detailed analysis of the blow-up behavior that $W_{0}^{1, p}$-capacities exhibit in the vicinity of the boundary of the underlying domain (cf. Lemma 3.4 and Theorem 3.6). 
In Section 4, we demonstrate that the capacities of the spaces $W^{1, p}(\Omega), W^{1, p}\left(\mathbb{R}^{d}\right)$, and $W_{0}^{1, p}\left(\Omega^{\prime}\right)$ are mutually equivalent on the power set $\mathcal{P}(\bar{\Omega})$. The proof of this equivalence is based on certain properties of extension operators and a localization argument.

Section 5 is concerned with the capacity of boundary sets. Here, we show that the capacities studied in Section 4 and the capacity of the trace space $W^{1-1 / p, p}(\partial \Omega)$ are equivalent on the power set $\mathcal{P}(\partial \Omega)$.

Lastly, in Section 6, we combine and summarize our findings. Here, we not only prove that the most common approaches to the sensitivity analysis of Signorini-type problems coincide (cf. Theorem 6.1, Corollary 6.6), but also state some general results on the fine properties of Sobolev functions (cf. Corollary 6.2, Corollary 6.3). The latter may also be of independent interest.

2. Capacity Theory in an Abstract Setting. In the following, we review basic results from capacity theory in an abstract setting that is tailored to the study of the spaces $W^{1, p}(\Omega), W^{1, p}\left(\mathbb{R}^{d}\right), W^{1-1 / p, p}(\partial \Omega)$, and $W_{0}^{1, p}\left(\Omega^{\prime}\right)$ considered in Sections 3 to 5 . The main results of this chapter concern the existence, the uniqueness, and the behavior of quasi continuous representatives, cf. Corollary 2.11, Lemma 2.12, and Theorem 2.13. Note that there are numerous different ways to introduce capacities (e.g., using kernel functions or distributions, cf. [1,2]). The approach that we employ in this paper utilizes the framework of topological spaces and is heavily inspired by the analysis in [12]. We consider the following situation:

Assumption 2.1 (Standing Assumptions and Notation for the Abstract Setting).

a) $(X, \mathcal{O}(X))$ is a topological space.

b) $\mathcal{B}(X)$ is the Borel $\sigma$-algebra on $(X, \mathcal{O}(X))$.

c) $\mathcal{P}(X)$ is the power set of $X$.

d) $\mu$ is a measure on $\mathcal{B}(X)$ with $\mu(A)>0$ for all $A \in \mathcal{O}(X) \backslash\{\emptyset\}$.

e) $1 \leq p<\infty$ is arbitrary but fixed.

f) $L^{p}(X, \mu)$ is the Lebesgue space of real-valued $(\mu, p)$-integrable functions.

g) $V \subseteq L^{p}(X, \mu)$ is a Banach space such that:

i) $V \cap C(X)$ is $\|\cdot\|_{V}$-dense in $V$,

ii) $\max (0, v) \in V$ and $\|\max (0, v)\|_{V} \leq\|v\|_{V}$ for all $v \in V$,

iii) there exists a constant $C>0$ with $\|v\|_{L^{p}} \leq C\|v\|_{V}$ for all $v \in V$.

For details on the topological concepts in Assumption 2.1, we refer to [25]. Note that, throughout this paper, the max-operator is always assumed to act pointwise $\mu$-almost everywhere. Using $V \subseteq L^{p}(X, \mu)$, we define the capacity of the function space $V$ as follows:

Definition 2.2 (Capacity). Given Assumption 2.1, the set function

$$
\begin{gathered}
\operatorname{cap}(\cdot ; X, V, \mu): \mathcal{P}(X) \rightarrow[0, \infty] \\
A \mapsto \inf \left\{\|v\|_{V}: v \geq 1 \mu \text {-a.e. in a nbhd. of } A\right\}
\end{gathered}
$$

is called the capacity generated by the triple $(X, V, \mu)$.

By a "neighborhood of $A$ " we of course mean a set $G \in \mathcal{O}(X)$ satisfying $A \subseteq G$. For the sake of brevity, we suppress the dependency on the triple $(X, V, \mu)$ in the rest of 
this section and simply write $\operatorname{cap}(\cdot)$ instead of $\operatorname{cap}(\cdot ; X, V, \mu)$. Some remarks are in order regarding Definition 2.2:

REMARK 2.3. In the theory of Sobolev and Dirichlet spaces, it is common to raise the term $\|v\|_{V}$ in the infimum on the right-hand side of (2.1) to a suitable power. If, e.g., $V$ is a Dirichlet space (and thus Hilbert), one typically defines the capacity to be equal to $\inf \left\{\|v\|_{V}^{2}: v \geq 1 \mu\right.$-a.e. in a nbhd. of $\left.A\right\}$ (cf. [12, Section 3.1]). Given the setting of Assumption 2.1, however, where no further information about the space $V$ and its norm $\|\cdot\|_{V}$ is available, such an approach is unnatural. Replacing the term $\|v\|_{V}$ in (2.1) with, e.g., $\|v\|_{V}^{p}$ would even cause the resulting capacity to be non-subadditive in general (cf. the proof of Lemma 2.5d) below). We emphasize that the equivalency estimates obtained in Sections 3 to 5 using Definition 2.2 can easily be transformed to conform to the definitions of Sobolev capacity employed in $[5,8,11,16]$ etc.

Since $\max (0, v) \in V$ and $\|\max (0, v)\|_{V} \leq\|v\|_{V}$ for all $v \in V$, we instantly obtain an alternative representation of the capacity.

Corollary 2.4. The capacity $\operatorname{cap}(\cdot)$ can equivalently be computed by

$\operatorname{cap}(A)=\inf \left\{\|v\|_{V}: v \geq 0 \mu\right.$-a.e. in $X, v \geq 1 \mu$-a.e. in a nbhd. of $\left.A\right\} \quad \forall A \subseteq X$.

By adapting the proofs in [16, Section 2] and [5, Section 5.8.2], we obtain the following:

Lemma 2.5 (Elementary Properties of the Capacity).

a) If $\operatorname{cap}(A)=0$ for $A \in \mathcal{B}(X)$, then $\mu(A)=0$ as well.

b) If $A_{1} \subseteq A_{2} \subseteq X$, then $\operatorname{cap}\left(A_{1}\right) \leq \operatorname{cap}\left(A_{2}\right)$.

c) If $A_{i}, i=1, \ldots, n$, is a finite collection of subsets of $X$, then

$$
\frac{1}{n} \sum_{i=1}^{n} \operatorname{cap}\left(A_{i}\right) \leq \operatorname{cap}\left(\bigcup_{i=1}^{n} A_{i}\right) \text {. }
$$

d) If $A_{i}, i \in \mathbb{N}$, is a countable collection of subsets of $X$, then

$$
\operatorname{cap}\left(\bigcup_{i=1}^{\infty} A_{i}\right) \leq \sum_{i=1}^{\infty} \operatorname{cap}\left(A_{i}\right) \text {. }
$$

Proof. Due to Assumption 2.1g), there exists a $C>0$ such that

$$
\begin{aligned}
0 \leq \frac{1}{C} \mu(A)^{1 / p} & \leq \frac{1}{C} \inf \left\{\|v\|_{L^{p}}: v \geq 1 \mu \text {-a.e. in a nbhd. of } A\right\} \\
& \leq \inf \left\{\|v\|_{V}: v \geq 1 \mu \text {-a.e. in a nbhd. of } A\right\}=\operatorname{cap}(A) \quad \forall A \in \mathcal{B}(X),
\end{aligned}
$$

immediately yielding part $a$ ). The monotonicity property in $b$ ) holds since the set of functions over which the infimum in the definition of $\operatorname{cap}\left(A_{2}\right)$ is taken is a subset of the set in the definition of $\operatorname{cap}\left(A_{1}\right)$. To obtain $c$ ), it sufficies to add up the inequalities $\operatorname{cap}\left(A_{i}\right) \leq \operatorname{cap}\left(\bigcup_{j=1}^{n} A_{j}\right), i=1, \ldots, n$. It remains to prove $\left.d\right)$. To this end, let $A_{i} \subseteq X$, $i \in \mathbb{N}$, be a countable collection of sets. We may assume w.l.o.g. that the series on the right-hand side of (2.2) is finite, otherwise the inequality holds trivially. Consider now an arbitrary but fixed $\varepsilon>0$. Then we obtain from the alternative representation of $\operatorname{cap}(\cdot)$ in Corollary 2.4 that for every $i \in \mathbb{N}$ we can find a $v_{i} \in V$ with $v_{i} \geq 1 \mu$-a.e. in a neighborhood of $A_{i}, v_{i} \geq 0 \mu$-a.e. in $X$, and

$$
\operatorname{cap}\left(A_{i}\right) \leq\left\|v_{i}\right\|_{V} \leq \operatorname{cap}\left(A_{i}\right)+\frac{\varepsilon}{2^{i}} .
$$


Since $V$ is Banach, we can define $v:=\sum_{i=1}^{\infty} v_{i} \in V$ and obtain

$$
v \geq 1 \mu \text {-a.e. in a nbhd. of } \bigcup_{i=1}^{\infty} A_{i}, \quad \operatorname{cap}\left(\bigcup_{i=1}^{\infty} A_{i}\right) \leq\|v\|_{V} \leq \varepsilon+\sum_{i=1}^{\infty} \operatorname{cap}\left(A_{i}\right) .
$$

Letting $\varepsilon \rightarrow 0$ in the above yields (2.2). This completes the proof.

Due to its construction, cf. Definition 2.2, the capacity cap $(\cdot)$ has just the right "detection sensitivity" that is needed to properly identify those sets in $\mathcal{P}(X)$ that are negligible in the study of the function space $V$. Analogously to the classical almost everywhere sense, we define:

Definition 2.6 ([12, Chapter 3]). If $A$ is a subset of $X$, then a statement depending on $x \in A$ is said to hold quasi everywhere (q.e.) in $A$ if there exists a set $N \subseteq X$ of zero capacity (a so-called polar set) such that the statement is true for all $x \in A \backslash N$.

Remark 2.7. Definition 2.2 implies that for every set $N \subseteq X$ with $\operatorname{cap}(N)=0$ we can find an $N^{\prime} \in \mathcal{B}(X)$ such that $N \subseteq N^{\prime}$ and $\operatorname{cap}\left(N^{\prime}\right)=0$. As a consequence, we may always assume w.l.o.g. that the exceptional set in Definition 2.6 is Borel measurable.

Note that the notion of q.e. is always at least as strict as the almost everywhere sense of the measure space $(X, \mathcal{B}(X), \mu)$ (cf. Lemma 2.5a) and Remark 2.7). The more regular the functions in the space $V$ are, the more restrictive the notion of quasi everywhere becomes. In the following, the (semi-)continuity of functions up to sets of capacity zero will be of particular importance for our analysis (cf. $[12,16])$ :

Definition 2.8. A function $v: X \rightarrow \mathbb{R} \cup\{ \pm \infty\}$ is called quasi (lower/upper semi-) continuous if there exists a sequence of sets $\left(G_{k}\right) \subseteq \mathcal{O}(X)$ such that for all $k \in \mathbb{N}$

$$
\begin{gathered}
G_{k+1} \subseteq G_{k}, \quad \operatorname{cap}\left(G_{k}\right)<\frac{1}{k}, \\
v: X \backslash G_{k} \rightarrow \mathbb{R} \cup\{ \pm \infty\} \text { is (lower/upper semi-) continuous. }
\end{gathered}
$$

The terms "(lower/upper semi-) continuous" are understood in the topological sense, see $[25$, Section $7 \mathrm{~K}]$ for details on this topic. Further, we assume subsets of topological spaces to be endowed with the subset topology throughout this paper.

Definition 2.9. A sequence of functions $v_{n}: X \rightarrow \mathbb{R}$ is said to converge quasi uniformly in $X$ to a function $v: X \rightarrow \mathbb{R}$ if there exist sets $\left(G_{k}\right) \subseteq \mathcal{O}(X)$ with

$$
G_{k+1} \subseteq G_{k}, \quad \operatorname{cap}\left(G_{k}\right)<\frac{1}{k}, \quad \text { and } \lim _{n \rightarrow \infty}\left(\sup _{x \in X \backslash G_{k}}\left|v_{n}(x)-v(x)\right|\right)=0 \quad \forall k \in \mathbb{N} .
$$

As an immediate consequence of Definition 2.2 and the properties of $V$, we obtain:

Lemma 2.10. Let $v_{n} \in V \cap C(X)$ be a $\|\cdot\|_{V}$-Cauchy sequence. Then there exists a subsequence $\left(v_{n_{k}}\right)$ such that the continuous representatives of $\left(v_{n_{k}}\right)$ converge quasi uniformly in $X$ to a quasi continuous and Borel measurable function $u: X \rightarrow \mathbb{R}$.

Note that the continuous representative of an element of $V \cap C(X)$ is indeed unique, cf. Assumption 2.1d). Lemma 2.10 is obtained completely analogously to the classical Egorov theorem (cf. [4, Lemma 2.19] and also [16, Theorem 4.3]). We recall the proof for the convenience of the reader: 
Proof. Since $v_{n} \in V \cap C(X)$ is Cauchy in $V$, there exists a subsequence (still denoted by $v_{n}$ ) such that

$$
\sum_{n=1}^{\infty} 2^{n}\left\|v_{n}-v_{n+1}\right\|_{V}<\infty
$$

The above implies that for every $k \in \mathbb{N}$ there exists an $N_{k}$ with

$$
\sum_{n=N_{k}}^{\infty} 2^{n}\left\|v_{n}-v_{n+1}\right\|_{V}<\frac{1}{2 k}
$$

We assume w.l.o.g. that $N_{k} \leq N_{k+1}$ for all $k$ and define

$$
E_{n}:=\left\{x \in X:\left|v_{n}(x)-v_{n+1}(x)\right|>2^{-n}\right\} .
$$

Due to the continuity of $\left|v_{n}-v_{n+1}\right|, E_{n}$ is in $\mathcal{O}(X)$ and

$$
\begin{aligned}
\operatorname{cap}\left(E_{n}\right) & \leq\left\|2^{n}\left|v_{n}-v_{n+1}\right|\right\|_{V} \\
& \leq 2^{n}\left(\left\|\max \left(0, v_{n}-v_{n+1}\right)\right\|_{V}+\left\|\min \left(0, v_{n}-v_{n+1}\right)\right\|_{V}\right) \\
& \leq 2^{n+1}\left\|v_{n}-v_{n+1}\right\|_{V} .
\end{aligned}
$$

Setting

$$
G_{k}:=\bigcup_{n=N_{k}}^{\infty} E_{n} \in \mathcal{O}(X)
$$

we obtain (cf. Lemma 2.5d))

$$
\operatorname{cap}\left(G_{k}\right) \leq \sum_{n=N_{k}}^{\infty} \operatorname{cap}\left(E_{n}\right) \leq 2 \sum_{n=N_{k}}^{\infty} 2^{n}\left\|v_{n}-v_{n+1}\right\|_{V}<\frac{1}{k}
$$

and for all $N_{k} \leq m_{1} \leq m_{2}$

$$
\sup _{x \in X \backslash G_{k}}\left|v_{m_{1}}(x)-v_{m_{2}}(x)\right| \leq \sum_{n=m_{1}}^{m_{2}} \sup _{x \in X \backslash G_{k}}\left|v_{n}(x)-v_{n+1}(x)\right| \leq \sum_{n=m_{1}}^{m_{2}} 2^{-n} \stackrel{m_{1} \rightarrow \infty}{\longrightarrow} 0 .
$$

Accordingly, $\left.v_{n}\right|_{X \backslash G_{k}} \in C\left(X \backslash G_{k}\right)$ is uniformly Cauchy, and we may deduce from the uniform limit theorem (see [24, Theorem 4.2.10]) that $v_{n} \rightarrow u_{k}$ uniformly in $X \backslash G_{k}$ for some $u_{k} \in C\left(X \backslash G_{k}\right)$. Note that for $k_{1} \geq k_{2}$ we have $N_{k_{1}} \geq N_{k_{2}}$ and, consequently, $G_{k_{1}} \subseteq G_{k_{2}}$. Therefore,

$$
u_{k_{1}}(x)=u_{k_{2}}(x) \quad \forall x \in X \backslash G_{k_{2}}
$$

for all $k_{1} \geq k_{2}$ and by putting

$$
N:=\bigcap_{k=1}^{\infty} G_{k}, \quad u(x):=\left\{\begin{array}{ll}
u_{k}(x) & \text { if } x \in X \backslash G_{k} \text { for some } k \\
0 & \text { if } x \in N
\end{array},\right.
$$

we get a well-defined function $u: X \rightarrow \mathbb{R}$. This $u$ is obviously quasi continuous and $v_{n} \rightarrow u$ quasi uniformly in $X$, according to its construction. Moreover, $u$ is Borel measurable as one can easily check using the representation in (2.3). 
Using Lemma 2.10, it is straightforward to prove:

Corollary 2.11. If $v_{n} \in V \cap C(X)$ is a sequence with $v_{n} \rightarrow v$ in $V$, then there exists a subsequence $\left(v_{n_{k}}\right)$ and a Borel measurable, quasi continuous function $\tilde{v}: X \rightarrow \mathbb{R}$ such that the continuous representatives of $v_{n_{k}}$ converge to $\tilde{v}$ quasi uniformly in $X$ and such that $v=\tilde{v} \mu$-a.e. in $X$.

Proof. From Lemma 2.10 it follows that there exists a subsequence $\left(v_{n_{k}}\right)$ such that the continuous representatives of $v_{n_{k}}$ converge quasi uniformly in $X$ to a quasi continuous, Borel measurable function $\tilde{v}: X \rightarrow \mathbb{R}$. In particular, this implies that $v_{n_{k}} \rightarrow \tilde{v}$ pointwise q.e. in $X$. Moreover, we know that q.e. implies $\mu$-a.e. (cf. Lemma 2.5a) and Remark 2.7), and from Assumption 2.1g) we readily obtain (after possibly passing over to another subsequence) that $v_{n_{k}} \rightarrow v \mu$-a.e. in $X$. Consequently, $v=\tilde{v} \mu$-a.e., proving the claim.

Since $V \cap C(X)$ is dense in $V$ (see Assumption 2.1g)), Corollary 2.11 especially implies that every $v \in V$ possesses a quasi continuous representative $\tilde{v}: X \rightarrow \mathbb{R}$, cf. Lusin's theorem in the classical theory. In order to prove that this representative is unique up to sets of capacity zero, we need:

Lemma 2.12. Let $u: X \rightarrow \mathbb{R} \cup\{ \pm \infty\}$ be a quasi upper semi-continuous function satisfying $u \geq 0 \mu$-a.e. in $X$. Then $u \geq 0$ q.e. in $X$ as well.

Proof. We proceed analogously to [8, Lemma 6.49]: Let $\left(G_{k}\right) \in \mathcal{O}(X)$ be a sequence of sets such that

$$
\begin{gathered}
G_{k+1} \subseteq G_{k}, \quad \operatorname{cap}\left(G_{k}\right)<\frac{1}{k}, \\
u: X \backslash G_{k} \rightarrow \mathbb{R} \cup\{ \pm \infty\} \text { is upper semi-continuous. }
\end{gathered}
$$

Then $\left\{x \in X \backslash G_{k}: u(x)<0\right\} \in \mathcal{O}\left(X \backslash G_{k}\right)$ for every $k \in \mathbb{N}$ and the definition of the subset topology yields that $\left\{x \in X \backslash G_{k}: u(x)<0\right\} \cup G_{k} \in \mathcal{O}(X)$. Now let $v \in V$ be an arbitrary function satisfying $v \geq 1 \mu$-a.e. in a neighborhood of $G_{k}$.

We know that $\mu(\{x \in X: u(x)<0\}=0, v$ therefore also satisfies $v \geq 1 \mu$-a.e. in $\left\{x \in X \backslash G_{k}: u(x)<0\right\} \cup G_{k} \in \mathcal{O}(X)$, and we may deduce from Definition 2.2:

$$
\operatorname{cap}(\{x \in X: u(x)<0\}) \leq \operatorname{cap}\left(\left\{x \in X \backslash G_{k}: u(x)<0\right\} \cup G_{k}\right) \leq \operatorname{cap}\left(G_{k}\right)<\frac{1}{k} .
$$

Letting $k \rightarrow \infty$ in the above yields the claim.

We point out that Lemma 2.12 is also a useful tool in the study of sets that are defined by pointwise constraints. Details on this topic can be found in Section 6. By combining the results obtained so far, we arrive at:

TheOREM 2.13. Every $v \in V$ admits a quasi continuous representative $\tilde{v}: X \rightarrow \mathbb{R}$ and this quasi continuous representative is unique up to sets of capacity zero.

Proof. The existence of a quasi continuous representative follows immediately from Corollary 2.11 and the density of $V \cap C(X)$ in $V$. It remains to prove uniqueness. To this end, let $\tilde{v}_{1}, \tilde{v}_{2}: X \rightarrow \mathbb{R}$ be two quasi continuous representatives of $v$. Then $\tilde{v}_{1}-\tilde{v}_{2}$ is quasi continuous with $\tilde{v}_{1}-\tilde{v}_{2}=0 \mu$-a.e. in $X$, and we may employ Lemma 2.12 to deduce that $\tilde{v}_{1}-\tilde{v}_{2}=0$ holds q.e. in $X$. This proves the claim.

Since quasi continuous representatives are unique up to sets of capacity zero, it makes sense to talk about the quasi everywhere behavior of a function $v \in V$ :

DeFInITION 2.14. A function $v \in V$ is said to satisfy a pointwise condition quasi everywhere in $X$ if the respective condition is satisfied quasi everywhere by all quasi continuous representatives of $v$. 
The quasi everywhere sense defined above provides the most natural setting for the study of pointwise (in)equalities involving elements of the space $V$. Contrary to the $\mu$-a.e.-sense, it takes the regularity of the underlying function space into account and thus allows, e.g., for an adequate study of the contact sets of solutions to Signoriniand obstacle-type variational inequalities in Sobolev spaces (cf. [15, 17, 21, 22]). It should be noted that the concept of capacity and the notion of quasi everywhere also give rise to the so-called fine topology and the theory of Choquet integration. For details on the latter topics, we refer to $[1,2,16]$.

3. Capacity Theory and Sobolev Spaces. Having recalled the results from capacity theory needed for our analysis, we now turn our attention to the main topic of this paper - the comparison of the different notions of Sobolev capacity found throughout the literature. Henceforth, we consider the following setting:

Assumption 3.1 (Standing Assumptions and Notation for the Sobolev Setting).

a) $d \geq 2$ and $1<p \leq d .^{1}$

b) $\Omega \subseteq \mathbb{R}^{d}$ is a bounded (strong) Lipschitz domain (see [11, Definition 4.4]).

c) $D \subseteq \partial \Omega$ is relatively open and nonempty.

d) $\Omega^{\prime} \subseteq \mathbb{R}^{d}$ is an open set satisfying $\bar{\Omega} \subset \Omega^{\prime}$.

e) $\mathcal{L}^{d}$ is the d-dimensional Lebesgue measure.

f) $\mathcal{H}^{d-1}$ is the $(d-1)$-dimensional Hausdorff measure (scaled as in [11]).

g) $W^{1, p}(\Omega)$ and $\|\cdot\|_{W^{1, p}}$ are defined as in [5, Definition 5.1.3].

h) $W_{0}^{1, p}(\Omega)$ is the closure of $C_{c}^{\infty}(\Omega)$ in $W^{1, p}(\Omega)$.

i) $W_{D}^{1, p}(\Omega)$ is the closure of $\left\{v \in C^{\infty}(\bar{\Omega}): \operatorname{supp}(v) \cap \bar{D}=\emptyset\right\}$ in $W^{1, p}(\Omega)$.

j) $W_{0}^{1, p}(\Omega)$ and $W_{D}^{1, p}(\Omega)$ are both endowed with the norm $\|\cdot\|_{W^{1, p}}$.

k) $\operatorname{tr}: W^{1, p}(\Omega) \rightarrow W^{1-1 / p, p}(\partial \Omega)$ is the usual trace operator.

l) $W^{1-1 / p, p}(\partial \Omega)$ is endowed with the norm

$$
\|v\|_{W^{1-1 / p, p}(\partial \Omega)}:=\inf _{w \in \operatorname{tr}^{-1}(v)}\|w\|_{W^{1, p}(\Omega)} .
$$

Note that Assumption 3.1a) excludes the cases where $W^{1, p}(\Omega)$ embedds into the function space $C(\bar{\Omega})$. If $W^{1, p}(\Omega) \hookrightarrow C(\bar{\Omega})$, then the only set of $W^{1, p}$-capacity zero is the empty set and the study of Sobolev capacities becomes somewhat academic. The capacities that we will be concerned with in the remainder of this paper are the following:

$$
\begin{gathered}
\operatorname{cap}_{\Omega}(\cdot):=\operatorname{cap}\left(\cdot ; \bar{\Omega}, W^{1, p}(\Omega), \mathcal{L}^{d}\right), \quad \operatorname{cap}_{\Omega, \mathrm{D}}(\cdot):=\operatorname{cap}\left(\cdot ; \bar{\Omega}, W_{D}^{1, p}(\Omega), \mathcal{L}^{d}\right), \\
\operatorname{cap}_{\mathbb{R}^{d}}(\cdot):=\operatorname{cap}\left(\cdot ; \mathbb{R}^{d}, W^{1, p}\left(\mathbb{R}^{d}\right), \mathcal{L}^{d}\right), \quad \operatorname{cap}_{\Omega^{\prime}, 0}(\cdot):=\operatorname{cap}\left(\cdot ; \overline{\Omega^{\prime}}, W_{0}^{1, p}\left(\Omega^{\prime}\right), \mathcal{L}^{d}\right), \\
\operatorname{cap}_{\partial \Omega}(\cdot):=\operatorname{cap}\left(\cdot ; \partial \Omega, W^{1-1 / p, p}(\partial \Omega), \mathcal{H}^{d-1}\right) .
\end{gathered}
$$

Regarding the triples $(X, V, \mu)$ appearing in (3.1), some remarks are in order:

\footnotetext{
${ }^{1}$ The majority of the results proved in the following sections also holds for $p=1$. We exclude this case here to avoid discussing the problems and peculiarities that arise in the context of Hardy's inequality and the inverse trace theorem when $W^{1,1}$-spaces are considered, cf. [10] and [20].
} 


\section{REMARK 3.2.}

a) We always use the Euclidean topology on $\mathbb{R}^{d}$ and the associated subset topologies on $\partial \Omega, \bar{\Omega}$, and $\overline{\Omega^{\prime}}$. Recall that for a strong, bounded Lipschitz domain $\Omega$, the subset topology on $\partial \Omega$ and the topology induced by the atlas of $\partial \Omega$ are exactly the same.

b) Due to the area formula (see, e.g., [11, Theorem 3.8]), the (d-1)-dimensional Hausdorff measure coincides with the surface measure $S$ on $\mathcal{B}(\partial \Omega)$ for any strong bounded Lipschitz domain $\Omega$, i.e.,

$$
\mathcal{H}^{d-1}(A)=\int_{A} \mathrm{~d} \mathcal{H}^{d-1}=\int_{A} \mathrm{~d} S=S(A) \quad \forall A \in \mathcal{B}(\partial \Omega) .
$$

In particular, this implies that $\mathcal{H}^{d-1}$ (more precisely, the restriction of $\mathcal{H}^{d-1}$ to the $\sigma$-algebra $\mathcal{B}(\partial \Omega)$ ) is indeed a measure on $\mathcal{B}(\partial \Omega)$.

c) The map $W^{1, p}\left(\Omega^{\prime}\right) \ni v \mapsto \max (0, v) \in W^{1, p}\left(\Omega^{\prime}\right)$ is well-defined and continuous for every open set $\Omega^{\prime} \subseteq \mathbb{R}^{d}$ and every $1<p<\infty$ (cf. [5, Section 5.8.1]), and

$$
\nabla(\max (0, v))=\mathbb{1}_{\{v>0\}} \nabla v \quad \text { and } \quad\|\max (0, v)\|_{W^{1, p}} \leq\|v\|_{W^{1, p}}
$$

for all $v \in W^{1, p}\left(\Omega^{\prime}\right)$. Further, the density of $C(\bar{\Omega}) \cap W^{1, p}(\Omega)$ in $W^{1, p}(\Omega)$ and the fact that the trace operator $\operatorname{tr}: W^{1, p}(\Omega) \rightarrow W^{1-1 / p, p}(\partial \Omega)$ admits a continuous linear right inverse $\eta: W^{1-1 / p, p}(\partial \Omega) \rightarrow W^{1, p}(\Omega)$ (cf. [14, Theorem 1.5.1.3.]) yields $\max (0, v) \in W^{1-1 / p, p}(\partial \Omega)$ for all $v \in W^{1-1 / p, p}(\partial \Omega)$ and

$$
\begin{aligned}
\|\max (0, v)\|_{W^{1-1 / p, p}} & =\inf _{w \in W^{1, p}(\Omega): \operatorname{tr} w=\max (0, v)}\|w\|_{W^{1, p}} \\
& \leq \inf _{w \in W^{1, p}(\Omega): \operatorname{tr} w=v}\|\max (0, w)\|_{W^{1, p}} \leq\|v\|_{W^{1-1 / p, p}} .
\end{aligned}
$$

d) Recall that the norm $\|\cdot\|_{W^{1-1 / p, p}(\partial \Omega)}$ in Assumption 3.1l) is equivalent to

$$
\|v\|=\left(\int_{\partial \Omega}|v|^{p} \mathrm{~d} \mathcal{H}^{d-1}+\int_{\partial \Omega} \int_{\partial \Omega} \frac{|v(x)-v(y)|^{p}}{|x-y|^{d+p-2}} \mathrm{~d} \mathcal{H}^{d-1}(x) \mathrm{d} \mathcal{H}^{d-1}(y)\right)^{1 / p}
$$

for $v \in W^{1-1 / p, p}(\partial \Omega)$ (cf. [14, Section 1.3.3]).

Using the observations in Remark 3.2 and other standard results from the theory of Sobolev spaces, it is easy to check that the triples $(X, V, \mu)$ in $(3.1)$ all satisfy the conditions in Assumption 2.1. Accordingly, the theory of Section 2 is applicable, and we may indeed talk about polar sets and quasi continuous representatives with respect to $\operatorname{cap}_{\Omega}, \operatorname{cap}_{\Omega^{\prime}, 0}, \operatorname{cap}_{\mathbb{R}^{\mathrm{d}}}, \operatorname{cap}_{\partial \Omega}$, and $\operatorname{cap}_{\Omega, \mathrm{D}}$. We point out that all of the latter five capacities can be encountered in the literature (most commonly raised to the power $p$, cf. Remark 2.3). The first one, cap $\Omega$, appears, e.g., in [17]. The second one, $\operatorname{cap}_{\Omega^{\prime}, 0}$, can be found in [6]. The third and the fifth one, $\operatorname{cap}_{\mathbb{R}^{d}}$ and cap $\Omega, \mathrm{D}$, are commonly used in the study of partial differential equations (cf. $[5,11,16])$. Lastly, the capacity of the trace space $W^{1-1 / p, p}(\partial \Omega)$, cap $\partial \Omega$, has been considered in [15, Example 6]. We begin our study of the relationship between the capacities in (3.1) with the following elementary result:

Proposition 3.3.

$$
\operatorname{cap}_{\Omega}(\bar{\Omega})<\infty, \quad \operatorname{cap}_{\Omega^{\prime}, 0}(\bar{\Omega})<\infty, \quad \operatorname{cap}_{\mathbb{R}^{\mathrm{d}}}(\bar{\Omega})<\infty, \quad \text { and } \quad \operatorname{cap}_{\partial \Omega}(\partial \Omega)<\infty .
$$


Proof. Let $\varphi \in C_{c}^{\infty}\left(\mathbb{R}^{d}\right)$ be a bump function satisfying $\varphi \equiv 1$ in a neighborhood of $\bar{\Omega}$ and $\operatorname{supp} \varphi \subset \Omega^{\prime}$. Then Definition 2.2 implies

$$
\begin{gathered}
\operatorname{cap}_{\Omega}(\bar{\Omega}) \leq\left\|\left.\varphi\right|_{\Omega}\right\|_{W^{1, p}(\Omega)}=\mathcal{L}^{d}(\Omega)^{1 / p} \\
\operatorname{cap}_{\partial \Omega}(\partial \Omega) \leq\left\|\left.\varphi\right|_{\partial \Omega}\right\|_{W^{1-1 / p, p}(\partial \Omega)}, \\
\operatorname{cap}_{\mathbb{R}^{d}}(\bar{\Omega}) \leq\|\varphi\|_{W^{1, p}\left(\mathbb{R}^{d}\right)}, \quad \text { and } \quad \operatorname{cap}_{\Omega^{\prime}, 0}(\bar{\Omega}) \leq\left\|\left.\varphi\right|_{\Omega^{\prime}}\right\|_{W^{1, p}\left(\Omega^{\prime}\right)} .
\end{gathered}
$$

This proves the claim.

Because of Proposition 3.3 and Lemma 2.5, we know that the capacities cap $\Omega$, $\operatorname{cap}_{\Omega^{\prime}, 0}$, $\operatorname{cap}_{\mathbb{R}^{d}}$, and cap $\partial \Omega$ define finite outer measures on the closure $\bar{\Omega}$ and the boundary $\partial \Omega$, respectively. Note that this is certainly untrue for $\operatorname{cap}_{\Omega, \mathrm{D}}$, $\operatorname{since}_{\operatorname{cap}_{\Omega, \mathrm{D}}}(A)=\infty$ for every $A \in \mathcal{P}(\bar{\Omega})$ with $A \cap \bar{D} \neq \emptyset$ (cf. Definition 2.2 ). The quantity $\operatorname{cap}_{\Omega, \mathrm{D}}(A)$ may even be infinite when the set $A$ does not intersect $\bar{D}$, as the following lemma shows:

Lemma 3.4. If $\bar{D}$ is a $(d-1)$-set in the sense of [10, Definition 4.1], then there exists a constant $C>0$ such that

$$
\left(\int_{A} \frac{1}{\operatorname{dist}(\cdot, D)^{p}} \mathrm{~d}^{d}\right)^{1 / p} \leq C \operatorname{cap}_{\Omega, \mathrm{D}}(A) \quad \forall A \in \mathcal{P}(\bar{\Omega}) .
$$

Proof. Let $v \in W_{D}^{1, p}(\Omega)$ be an arbitrary but fixed function satisfying $v \geq 1 \mathcal{L}^{d}$-a.e. in a (relative) neighborhood of $A$. Then Hardy's inequality [10, Theorem 3.2] implies

$$
\int_{A} \frac{1}{\operatorname{dist}(x, D)^{p}} d \mathcal{L}^{d}(x) \leq \int_{\Omega} \frac{|v(x)|^{p}}{\operatorname{dist}(x, D)^{p}} d \mathcal{L}^{d}(x) \leq C\|v\|_{W^{1, p}(\Omega)}^{p} .
$$

Taking the infimum over all $v$ in (3.5) yields (3.4) as claimed.

Lemma 3.4 and [10, Theorem 3.4] immediately yield that $\operatorname{cap}_{\Omega, \mathrm{D}}(\Omega)=\infty$ whenever $\bar{D}$ is a $(d-1)$-set. Moreover, using Fatou's lemma, we may deduce from (3.4) that $\operatorname{cap}_{\Omega, \mathrm{D}}\left(A_{k}\right) \rightarrow \infty$ as $k \rightarrow \infty$ for every compact exhaustion $\left(A_{k}\right)_{k \in \mathbb{N}}$ of $\Omega$ and every $1<p \leq d$. This implies that $\operatorname{cap}_{\Omega, \mathrm{D}}$ cannot be equivalent to any of the other capacities in (3.1), i.e., there cannot exist constants $C_{1}, C_{2}>0$ with, e.g.,

$$
C_{1} \operatorname{cap}_{\Omega}(A) \leq \operatorname{cap}_{\Omega, \mathrm{D}}(A) \leq C_{2} \operatorname{cap}_{\Omega}(A) \quad \forall A \in \mathcal{P}(\Omega)(\text { or } \mathcal{P}(\bar{\Omega})) .
$$

Instead, the following type of equivalency estimate can be obtained:

Proposition 3.5. For all $A \in \mathcal{P}(\bar{\Omega})$

$$
\operatorname{cap}_{\Omega}(A) \leq \operatorname{cap}_{\Omega, \mathrm{D}}(A) \leq\left(1+\frac{d^{1 / p}}{\operatorname{dist}(A, D)}\right) \operatorname{cap}_{\Omega}(A)
$$

where

$$
\operatorname{dist}(A, D):=\inf _{x \in A, y \in D}|x-y| .
$$

Proof. The first inequality in (3.7) is trivial since $W_{D}^{1, p}(\Omega)$ is a subset of $W^{1, p}(\Omega)$ and since the capacities $\operatorname{cap}_{\Omega}$ and $\operatorname{cap}_{\Omega, \mathrm{D}}$ are both defined w.r.t. the subset topology of the closure $\bar{\Omega}$ (cf. Definition 2.2 and (3.1)). It remains to prove the second estimate. To this end, let $A \in \mathcal{P}(\bar{\Omega})$ be arbitrary but fixed. Note that (3.7) is trivially true 
if $\operatorname{dist}(A, D)=0$, so we may assume w.l.o.g. that $\operatorname{dist}(A, D)>0$. For small $\varepsilon>0$ consider

$$
\delta_{\varepsilon}: \bar{\Omega} \rightarrow[0,1], \quad \delta_{\varepsilon}(x):=\min \left(\max \left(0,(1+2 \varepsilon) \frac{\operatorname{dist}(x, D)}{\operatorname{dist}(A, D)}-\varepsilon\right), 1\right),
$$

and let $v \in W^{1, p}(\Omega)$ be an arbitrary function satisfying $v \geq 1 \mathcal{L}^{d}$-a.e. in a (relative) neighborhood of $A$. Then $\delta_{\varepsilon}$ is globally Lipschitz and $\delta_{\varepsilon} \equiv 0$ in a neighborhood of $\bar{D}$ as well as $\delta_{\varepsilon} \equiv 1$ in a neighborhood of $\bar{A}$. The latter implies that $v \delta_{\varepsilon}$ is an element of $W_{D}^{1, p}(\Omega)$ and that $v \delta_{\varepsilon} \geq 1 \mathcal{L}^{d}$-a.e. in a neighborhood of $A$. Further,

$$
\left\|v \delta_{\varepsilon}\right\|_{W^{1, p}} \leq\left(1+d^{1 / p}\left\|\nabla \delta_{\varepsilon}\right\|_{L^{\infty}}\right)\|v\|_{W^{1, p}} \leq\left(1+d^{1 / p} \frac{1+2 \varepsilon}{\operatorname{dist}(A, D)}\right)\|v\|_{W^{1, p}} .
$$

If we take the infimum over all $v$ in (3.8), then we obtain

$$
\operatorname{cap}_{\Omega, \mathrm{D}}(A) \leq\left(1+d^{1 / p} \frac{1+2 \varepsilon}{\operatorname{dist}(A, D)}\right) \operatorname{cap}_{\Omega}(A) \quad \forall \varepsilon>0 .
$$

Passing to the limit $\varepsilon \rightarrow 0$ in the above yields the claim.

Note that from (3.3), Lemma 3.4, and Proposition 3.5 it follows that, if $\bar{D}$ is a $(d-1)$ set, then there exist constants $C_{1}, C_{2}>0$ with

$$
C_{1}\left(\int_{A} \frac{1}{\operatorname{dist}(\cdot, D)^{p}} \mathrm{~d} \mathcal{L}^{d}\right)^{1 / p} \leq \operatorname{cap}_{\Omega, \mathrm{D}}(A) \leq C_{2}\left(1+\frac{d^{1 / p}}{\operatorname{dist}(A, D)}\right) \quad \forall A \in \mathcal{P}(\bar{\Omega}) .
$$

This shows that the qualitative behavior of the $W_{D}^{1, p}(\Omega)$-capacity is directly related to that of the distance function $A \ni x \mapsto \operatorname{dist}(x, D) \in[0, \infty)$. We point out that the second estimate in (3.9) is not optimal since there exist sets $A \subseteq \bar{\Omega}$ with $\operatorname{dist}(A, D)=0$ and $\operatorname{cap}_{\Omega, \mathrm{D}}(A)<\infty$. Studying the geometry of sets with the latter two properties is a very interesting field in itself. We will not go into details regarding this topic here but only prove the following exemplary result that gives an idea of what a set $A$ has to look like to obtain such a situation.

Theorem 3.6. Let $Q:=(0,1) \times(0,1)$ and $D:=(0,1) \times\{0\}$. Then for the sets

$$
A_{\alpha}:=\left\{(x, y) \in Q: x^{\alpha}<y\right\}, \quad \alpha>0,
$$

the following holds:

a) $A_{\alpha}$ is open and $\operatorname{dist}\left(A_{\alpha}, D\right)=0$ for all $\alpha>0$.

b) For $1<p<2$ :

$$
\operatorname{cap}_{Q, D}\left(A_{\alpha}\right)=\operatorname{cap}\left(A_{\alpha} ; \bar{Q}, W_{D}^{1, p}(Q), \mathcal{L}^{2}\right)\left\{\begin{array}{ll}
<\infty & \text { if } \alpha<\frac{1}{p-1} \\
=\infty & \text { if } \alpha \geq \frac{1}{p-1}
\end{array} .\right.
$$

c) For $p=2$ :

$$
\operatorname{cap}_{Q, D}\left(A_{\alpha}\right)=\operatorname{cap}\left(A_{\alpha} ; \bar{Q}, W_{D}^{1,2}(Q), \mathcal{L}^{2}\right)=\infty \text { for all } \alpha>0 .
$$


Proof. Part $a$ ) is easy to see. To obtain $b$ ), we proceed in several steps: Firstly, we note that $\bar{D}=[0,1] \times\{0\}$ obviously is a 1-set and that, according to Lemma 3.4, for all $1<p \leq 2$ there exists a constant $C>0$ with

$$
C \operatorname{cap}_{Q, D}\left(A_{\alpha}\right)^{p} \geq \int_{A_{\alpha}} \frac{1}{\operatorname{dist}(\cdot, D)^{p}} \mathrm{~d} \mathcal{L}^{2}=\int_{0}^{1} \int_{0}^{y^{1 / \alpha}} \frac{1}{y^{p}} \mathrm{~d} x \mathrm{~d} y=\int_{0}^{1} y^{-p+1 / \alpha} \mathrm{d} y
$$

This implies $\operatorname{cap}_{Q, D}\left(A_{\alpha}\right)=\infty$ for all $\alpha \geq 1 /(p-1)$ and proves the second case in (3.10). It remains to show that $\operatorname{cap}_{Q, D}\left(A_{\alpha}\right)<\infty$ for $0<\alpha<1 /(p-1)$ and $1<p<2$. To this end, for the time being, assume that $p-1<\alpha<1 /(p-1)$ and define

$$
v_{\alpha}: Q \rightarrow \mathbb{R}, \quad v_{\alpha}(x, y):=\min \left(1, \frac{y}{x^{\alpha}}\right) .
$$

Then $v_{\alpha}$ is in $L^{\infty}(Q) \cap W_{l o c}^{1, \infty}(Q)$ and in the distributional sense

$$
\left(\nabla v_{\alpha}\right)(x, y)= \begin{cases}\left(-\alpha \frac{y}{x^{\alpha+1}}, \frac{1}{x^{\alpha}}\right) & \mathcal{L}^{2} \text {-a.e. in }\left\{(x, y) \in Q: y<x^{\alpha}\right\} \\ (0,0) & \mathcal{L}^{2} \text {-a.e. in }\left\{(x, y) \in Q: y \geq x^{\alpha}\right\}\end{cases}
$$

From the above, we obtain by straightforward calculation

$$
\int_{Q}\left|\partial_{x} v_{\alpha}\right|^{p}+\left|\partial_{y} v_{\alpha}\right|^{p} \mathrm{~d} \mathcal{L}^{2}=\int_{0}^{1} \frac{\alpha^{p}}{p+1} x^{\alpha-p}+x^{\alpha(1-p)} \mathrm{d} x<\infty
$$

i.e., $v_{\alpha} \in W^{1, p}(Q)$. Further, our construction yields $v_{\alpha} \geq 1 \mathcal{L}^{2}$-a.e. in $A_{\alpha}$ and $\operatorname{tr} v_{\alpha}=0$ $\mathcal{H}^{1}$-a.e. on $D$, where the latter follows from the continuity of $v_{\alpha}$ on $\bar{Q} \backslash\{0\}$, the properties of the trace operator, and a localization argument. Since $A_{\alpha}$ is open, we directly obtain

$$
v_{\alpha} \in\left\{v \in W_{D}^{1, p}(Q): v \geq 1 \mathcal{L}^{2} \text {-a.e. in a nbhd. of } A_{\alpha}\right\} \neq \emptyset .
$$

Accordingly, $\operatorname{cap}_{Q, D}\left(A_{\alpha}\right)<\infty$ and the second case in (3.10) is proven for all $1<p<2$ and $(p-1)<\alpha<1 /(p-1)$. For the remaining $\alpha$, (3.10) follows from the monotonicity of $\operatorname{cap}_{Q, D}$ (cf. Lemma 2.5b)) and the fact that $A_{\alpha_{1}} \subseteq A_{\alpha_{2}}$ for all $0<\alpha_{1} \leq \alpha_{2}$. This completes the proof of $b$ ).

It remains to show that $\operatorname{cap}_{Q, D}\left(A_{\alpha}\right)=\infty$ for all $\alpha>0$ in the case $p=2$. Note that the latter is already proven for $\alpha \geq 1$ (see (3.11)), so we may restrict our analysis to the case $\alpha \in(0,1)$, which we prove by contradiction. Assuming the existence of an $\alpha \in(0,1)$ with $\operatorname{cap}_{Q, D}\left(A_{\alpha}\right)<\infty$, we can find at least one function $v \in W_{D}^{1,2}(Q)$ satisfying $v \geq 1 \mathcal{L}^{2}$-a.e. in a neighborhood of $A_{\alpha}$. Define $P:=\left\{(x, y) \in Q: y<x^{\alpha}\right\}$ and let $w:=\left.\min (1, v)\right|_{P}$. Then $P$ is a Lipschitz domain, and it follows from Remark 3.2c) and our construction that $w \in W^{1,2}(P)$ with $\operatorname{tr} w \equiv 0$ on $(0,1) \times\{0\}$ and $\operatorname{tr} w \equiv 1$ on $\left\{\left(x, x^{\alpha}\right): x \in(0,1)\right\}$. A function that is locally a step function, however, cannot be an element of $H^{1 / 2}(\partial P)$ (as one can easily calculate using the Sobolev-Slobodeckij norm (3.2)). This contradiction allows us to deduce that the set $\left\{v \in W_{D}^{1,2}(Q): v \geq 1 \mathcal{L}^{2}\right.$-a.e. in a nbhd. of $\left.A_{\alpha}\right\}$ is empty for all $\alpha>0$, which yields c) and completes the proof of the theorem. 


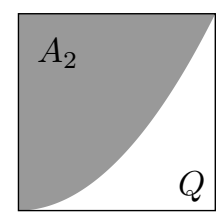

FIG. 3.1. $\alpha=2$

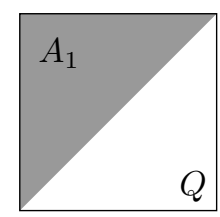

FIG. 3.2. $\alpha=1$

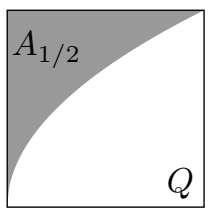

FIG. 3.3. $\alpha=1 / 2$

REMARK 3.7. Theorem 3.6 not only demonstrates that there exist situations with $\operatorname{dist}(A, D)=0$ and $\operatorname{cap}_{\Omega, \mathrm{D}}(A)<\infty$, but also shows that the capacities $\operatorname{cap}_{\Omega, \mathrm{D}}$ are typically nonequivalent (in the sense of (3.6)) for different values of $p$.

The singular behavior exhibited by the set function $\operatorname{cap}_{\Omega, \mathrm{D}}$ on and near the Dirichlet boundary part $D$ is the main reason why $W_{D}^{1, p}(\Omega)$-capacities (and, consequently, the $W_{0}^{1, p}(\Omega)$-capacity as a special case) are unfit for applications that require an adequate study of the boundary $\partial \Omega$. Note that in contrast to $\operatorname{cap}_{\Omega, \mathrm{D}}$, the capacities cap $\Omega$, $\operatorname{cap}_{\Omega^{\prime}, 0}, \operatorname{cap}_{\mathbb{R}^{d}}$, and cap $\partial \Omega$ are all able to meaningfully measure subsets of $\partial \Omega$, and, as we have mentioned in the introduction, all of these capacities have been used at one point or another in the literature as a substitute for $\operatorname{cap}_{\Omega, 0}$. In what follows, we will show that the latter four capacities are, in fact, equivalent on $\mathcal{P}(\partial \Omega)$ and give rise to the same quasi everywhere sense on the boundary $\partial \Omega$. We begin our investigation by studying the capacities $\operatorname{cap}_{\Omega}$, $\operatorname{cap}_{\Omega^{\prime}, 0}$, and $\operatorname{cap}_{\mathbb{R}^{\mathrm{d}}}$ on the closure $\bar{\Omega}$.

4. Equivalence of $\operatorname{cap}_{\Omega}, \operatorname{cap}_{\Omega^{\prime}, 0}$, and $\operatorname{cap}_{\mathbb{R}^{\mathbf{d}}}$ on $\overline{\boldsymbol{\Omega}}$. The first step in our study of the capacities $\operatorname{cap}_{\Omega}$, $\operatorname{cap}_{\Omega^{\prime}, 0}$, and $\operatorname{cap}_{\mathbb{R}^{\mathrm{d}}}$ is the following result:

LEMMA 4.1.

$$
\operatorname{cap}_{\Omega}(A) \leq \operatorname{cap}_{\mathbb{R}^{\mathrm{d}}}(A) \leq \operatorname{cap}_{\Omega^{\prime}, 0}(A) \quad \forall A \in \mathcal{P}(\bar{\Omega})
$$

Proof. Using restriction, extension by zero, and the definitions of the subset topologies on $\overline{\Omega^{\prime}}$ and $\bar{\Omega}$, we obtain that for all $A \subseteq \bar{\Omega}$ :

$$
\begin{aligned}
& \operatorname{cap}_{\Omega}(A) \\
& =\inf \left\{\|v\|_{W^{1, p}(\Omega)}: v \in W^{1, p}(\Omega), \exists G \in \mathcal{O}\left(\mathbb{R}^{d}\right) \text { s.t. } A \subseteq G \text { and } v \geq 1 \mathcal{L}^{d} \text {-a.e. in } G \cap \bar{\Omega}\right\} \\
& \leq \inf \left\{\left\|\left.v\right|_{\Omega}\right\|_{W^{1, p}(\Omega)}: v \in W^{1, p}\left(\mathbb{R}^{d}\right), \exists G \in \mathcal{O}\left(\mathbb{R}^{d}\right) \text { s.t. } A \subseteq G \text { and } v \geq 1 \mathcal{L}^{d} \text {-a.e. in } G\right\} \\
& \leq \inf \left\{\|v\|_{W^{1, p}\left(\mathbb{R}^{d}\right)}: v \in W^{1, p}\left(\mathbb{R}^{d}\right), \exists G \in \mathcal{O}\left(\mathbb{R}^{d}\right) \text { s.t. } A \subseteq G \text { and } v \geq 1 \mathcal{L}^{d} \text {-a.e. in } G\right\} \\
& =\operatorname{cap}_{\mathbb{R}^{d}}(A) \\
& \leq \inf \left\{\|v\|_{W^{1, p}\left(\Omega^{\prime}\right)}: v \in W_{0}^{1, p}\left(\Omega^{\prime}\right), \exists G \in \mathcal{O}\left(\mathbb{R}^{d}\right) \text { s.t. } A \subseteq G \text { and } v \geq 1 \mathcal{L}^{d} \text {-a.e. in } G\right\} \\
& =\inf \left\{\|v\|_{W^{1, p}\left(\Omega^{\prime}\right)}: v \in W_{0}^{1, p}\left(\Omega^{\prime}\right), \exists G \in \mathcal{O}\left(\mathbb{R}^{d}\right) \text { s.t. } A \subseteq G \text { and } v \geq 1 \mathcal{L}^{d} \text {-a.e. in } G \cap \Omega^{\prime}\right\} \\
& =\operatorname{cap}_{\Omega^{\prime}, 0}(A) .
\end{aligned}
$$

This yields the claim.

To prove that the capacities $\operatorname{cap}_{\Omega}$, $\operatorname{cap}_{\mathbb{R}^{\mathrm{d}}}$, and $\operatorname{cap}_{\Omega^{\prime}, 0}$ are equivalent on $\bar{\Omega}$, it remains to show that there exists a constant $C>0$ with

$$
\operatorname{cap}_{\Omega^{\prime}, 0}(A) \leq C \operatorname{cap}_{\Omega}(A) \quad \forall A \in \mathcal{P}(\bar{\Omega}) .
$$

Unfortunately, the derivation of (4.2) is not as straightforward as that of (4.1): The proof of Lemma 4.1 is comparatively simple because the restriction $\left.v\right|_{\Omega}$ of a function 
$v$ satisfying $v \geq 1$ a.e. on an $\mathbb{R}^{d}$ - or $\overline{\Omega^{\prime}}$-open set always satisfies $\left.v\right|_{\Omega} \geq 1$ a.e. on an $\bar{\Omega}$-open set (cf. the definition of the subset topology). To prove (4.2), we have to recover the condition " $v \geq 1$ a.e. in an $\overline{\Omega^{\prime}}$-neighborhood" from the condition " $v \geq 1$ a.e. in an $\bar{\Omega}$-neighborhood", i.e., we have to handle the transition from the subset topology of $\bar{\Omega}$ to the topology of the ambient space $\overline{\Omega^{\prime}}$, which is nontrivial.

In what follows, we will first prove (4.2) in a prototypical situation and afterwards use localization and rectification arguments to obtain the equivalency estimate in the general case.

Lemma 4.2. Let $B(s)$ denote the open ball in $\mathbb{R}^{d-1}$ with radius $s$ centered at the origin and let

$$
U(s, t):=B(s) \times(-t, t), \quad V(s, t):=B(s) \times(0, t), \quad W(s, t):=B(s) \times[0, t) .
$$

Then for all $r, \varepsilon>0$ there exists a constant $C=C(r, \varepsilon)$ with

$$
\operatorname{cap}_{U(3 r, 3 \varepsilon), 0}(A) \leq C \operatorname{cap}_{V(3 r, 3 \varepsilon)}(A) \quad \forall A \in \mathcal{P}(W(r, \varepsilon)) .
$$

Proof. Let $r, \varepsilon>0$ and $A \subseteq W(r, \varepsilon)$ be arbitrary but fixed. Assume that a function $v \in W^{1, p}(V(3 r, 3 \varepsilon))$ and an open set $G \in \mathcal{O}\left(\mathbb{R}^{d}\right)$ are given such that

$$
A \subseteq G \text { and } \quad v \geq 1 \mathcal{L}^{d} \text {-a.e. in } G \cap \overline{V(3 r, 3 \varepsilon)} .
$$

From this $v$ we construct a $u \in W_{0}^{1, p}(U(3 r, 3 \varepsilon))$ with $u \geq 1$ in a $U(3 r, 3 \varepsilon)$-neighborhood of $A$ and $\|u\|_{W^{1, p}(U(3 r, 3 \varepsilon))} \leq C(r, \varepsilon)\|v\|_{W^{1, p}(V(3 r, 3 \varepsilon))}$. To this end, let $\psi \in C_{c}^{\infty}\left(\mathbb{R}^{d}\right)$ be an arbitrary but fixed bump function satisfying

$$
\psi \equiv 1 \text { in } U(r, \varepsilon), \quad \psi \in[0,1] \text { in } U(2 r, 2 \varepsilon) \backslash U(r, \varepsilon), \quad \text { and } \quad \psi \equiv 0 \text { in } \mathbb{R}^{d} \backslash U(2 r, 2 \varepsilon) .
$$

Then $\psi v$ satisfies

$$
\begin{gathered}
\psi v \in W^{1, p}(V(3 r, 3 \varepsilon)), \quad \psi v=0 \mathcal{L}^{d} \text {-a.e. in } V(3 r, 3 \varepsilon) \backslash V(2 r, 2 \varepsilon), \\
\psi v=v \geq 1 \mathcal{L}^{d} \text {-a.e. in } G \cap V(r, \varepsilon), \quad A \subseteq G \cap W(r, \varepsilon) .
\end{gathered}
$$

We define

$$
u(x):= \begin{cases}(\psi v)(x) & \text { if } x \in V(3 r, 3 \varepsilon) \\ (\psi v)\left(x_{1}, \ldots, x_{d-1},-x_{d}\right) & \text { if }\left(x_{1}, \ldots, x_{d-1},-x_{d}\right) \in V(3 r, 3 \varepsilon)\end{cases}
$$

so that

$$
\begin{gathered}
u \in W^{1, p}(U(3 r, 3 \varepsilon)), \quad u=0 \mathcal{L}^{d} \text {-a.e. in } U(3 r, 3 \varepsilon) \backslash U(2 r, 2 \varepsilon), \\
\|u\|_{W^{1, p}(U(3 r, 3 \varepsilon))} \leq 2\|\psi v\|_{W^{1, p}(V(3 r, 3 \varepsilon))}
\end{gathered}
$$

and $u \geq 1 \mathcal{L}^{d}$-a.e. in

$$
H:=(G \cap W(r, \varepsilon)) \cup\left\{x \in \mathbb{R}^{d}:\left(x_{1}, \ldots, x_{d-1},-x_{d}\right) \in G \cap W(r, \varepsilon)\right\} \subseteq U(r, \varepsilon) .
$$

Note that from our construction it follows that $A$ is a subset of $H$. Further, $H$ is open. To see the latter, one can argue as follows:

Suppose that there exists an $x \in H$ such that there is no $\delta$-ball around $x$ that is contained in $H$. Then we can find a sequence $\left(x_{n}\right) \subseteq U(r, \varepsilon) \backslash H$ with $x_{n} \rightarrow x$, and one of the following has to be the case: 
1. There exists a subsequence of $x_{n}$ (unrelabeled) contained in $W(r, \varepsilon)$. In this case, $x \in G \cap W(r, \varepsilon)$ and

$$
x_{n} \in W(r, \varepsilon) \backslash H=W(r, \varepsilon) \backslash(G \cap W(r, \varepsilon)) \subseteq U(r, \varepsilon) \backslash(G \cap U(r, \varepsilon)) .
$$

But $x \in G \cap U(r, \varepsilon)$ and the set $G \cap U(r, \varepsilon)$ is open. This is a contradiction.

2. There exists a subsequence of $x_{n}$ (unrelabeled) with $x_{n} \in B(r) \times(-\varepsilon, 0]$. In this case, the sequence that is obtained by reflecting $x_{n}$ along the hyperplane $\mathbb{R}^{d-1} \times\{0\}$ has the properties in 1 ., and we again get a contradiction.

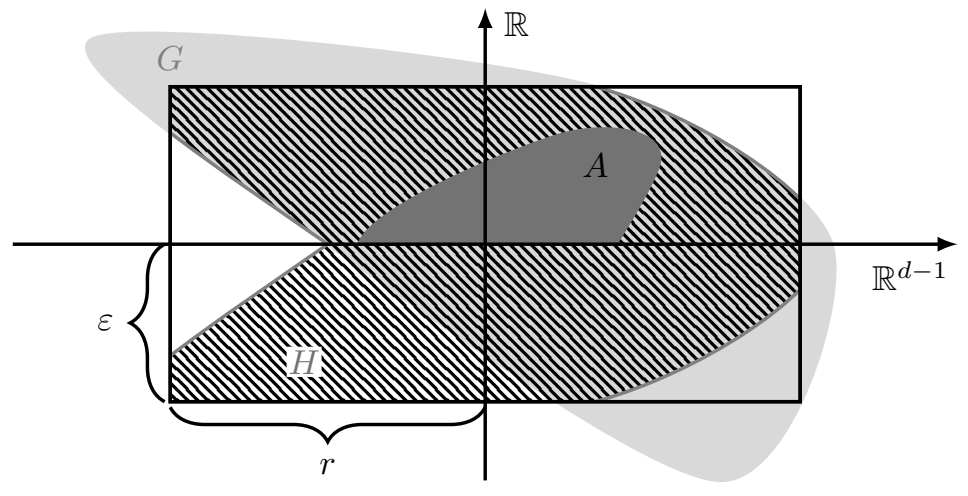

FIG. 4.1. Construction of the neighborhood $H$ in the proof of Lemma 4.2.

We now arrive at the following situation

$$
\begin{gathered}
u \in W_{0}^{1, p}(U(3 r, 3 \varepsilon)), \\
\|u\|_{W^{1, p}(U(3 r, 3 \varepsilon))} \leq 2\|\psi v\|_{W^{1, p}(V(3 r, 3 \varepsilon))}, \\
u \geq 1 \mathcal{L}^{d} \text {-a.e. in } H, \quad H \subseteq U(3 r, 3 \varepsilon) \text { open, } \quad A \subseteq H .
\end{gathered}
$$

Using the above and taking the infimum over all $v \in W^{1, p}(V(3 r, 3 \varepsilon))$ satisfying (4.3) for some open set $G$, we obtain

$$
\begin{aligned}
& \operatorname{cap}_{U(3 r, 3 \varepsilon), 0}(A) \\
& \quad=\inf \left\{\|u\|_{W^{1, p}}: u \in W_{0}^{1, p}(U(3 r, 3 \varepsilon)), u \geq 1 \mathcal{L}^{d} \text {-a.e. in a } \overline{U(3 r, 3 \varepsilon)} \text {-nbhd. of } A\right\} \\
& \quad \leq \inf \left\{2\|\psi v\|_{W^{1, p}}: v \in W^{1, p}(V(3 r, 3 \varepsilon)), v \geq 1 \mathcal{L}^{d} \text {-a.e. in a } \overline{V(3 r, 3 \varepsilon)} \text {-nbhd. of } A\right\} \\
& \quad \leq C(\psi) \operatorname{cap}_{V(3 r, 3 \varepsilon)}(A)
\end{aligned}
$$

with a constant $C=C(\psi)=C(r, \varepsilon)$.

To reduce the general case to the special situation studied in Lemma 4.2, we need the following result on the Lipschitz stability of $W^{1, p_{\text {- }}}$ and $W_{0}^{1, p}$-capacities:

Lemma 4.3. Let $\Omega_{1}, \Omega_{2} \subseteq \mathbb{R}^{d}$ be bounded strong Lipschitz domains, and let $\Omega_{1}^{\prime}, \Omega_{2}^{\prime}$ be open sets satisfying $\overline{\Omega_{i}} \subseteq \Omega_{i}^{\prime}, i=1,2$. Assume that $\Phi: \Omega_{1}^{\prime} \rightarrow \Omega_{2}^{\prime}$ is a bi-Lipschitz mapping with $\Phi\left(\overline{\Omega_{1}}\right)=\overline{\Omega_{2}}$. Then there exist constants $c, C>0$ depending only on $\Phi$ such that

$$
v \circ \Phi \in W^{1, p}\left(\Omega_{1}\right), \quad\|v \circ \Phi\|_{W^{1, p}\left(\Omega_{1}\right)} \leq C\|v\|_{W^{1, p}\left(\Omega_{2}\right)}
$$

for every $v \in W^{1, p}\left(\Omega_{2}\right)$ and

$$
\begin{gathered}
c \operatorname{cap}_{\Omega_{1}}\left(\Phi^{-1}(A)\right) \leq \operatorname{cap}_{\Omega_{2}}(A) \leq C \operatorname{cap}_{\Omega_{1}}\left(\Phi^{-1}(A)\right), \\
c \operatorname{cap}_{\Omega_{1}, 0}\left(\Phi^{-1}(A)\right) \leq \operatorname{cap}_{\Omega_{2}, 0}(A) \leq C \operatorname{cap}_{\Omega_{1}, 0}\left(\Phi^{-1}(A)\right)
\end{gathered}
$$


for all $A \subseteq \overline{\Omega_{2}}$.

Proof. The $W^{1, p}$-regularity of $v \circ \Phi$ and the estimate $\|v \circ \Phi\|_{W^{1, p}\left(\Omega_{1}\right)} \leq C\|v\|_{W^{1, p}\left(\Omega_{2}\right)}$ are standard results that can be found in, e.g., [26, Theorem 2.2.2]. The inequalities (4.6) and (4.7) follow immediately from (4.5), the definitions of the involved $W^{1, p_{-}}$ capacities, and the fact that $v \mapsto v \circ \Phi$ maps $W_{0}^{1, p}\left(\Omega_{2}\right)$ into $W_{0}^{1, p}\left(\Omega_{1}\right)$.

We are now in the position to prove (4.2):

Proposition 4.4. There exists a constant $C=C\left(\Omega, \Omega^{\prime}\right)>0$ such that

$$
\operatorname{cap}_{\Omega^{\prime}, 0}(A) \leq C \operatorname{cap}_{\Omega}(A) \quad \forall A \in \mathcal{P}(\bar{\Omega}) .
$$

Proof. Recall that, according to the definition of a strong Lipschitz domain (see [11, Definition 4.4]), for all $q \in \partial \Omega$ there exist an orthogonal transformation $R_{q} \in O(d)$, an open ball $B_{q} \subset \mathbb{R}^{d-1}$ with midpoint $x_{q} \in \mathbb{R}^{d-1}$, an open interval $J_{q}=\left(a_{q}, b_{q}\right)$, and a Lipschitz map $h_{q}: B_{q} \rightarrow J_{q}$ such that

$$
q \in R_{q}\left(B_{q} \times J_{q}\right) \quad \text { and } \quad \Omega \cap R_{q}\left(B_{q} \times J_{q}\right)=R_{q}\left(\left\{(x, y): x \in B_{q}, h_{q}(x)<y<b_{q}\right\}\right) \text {. }
$$

Note that, by making the sets $J_{q}$ and $B_{q}$ smaller, in the above situation we can always obtain that $\overline{R_{q}\left(B_{q} \times J_{q}\right)}$ is a subset of $\Omega^{\prime}$ and that

$$
\left\{(x, y):\left\|x-x_{q}\right\|<4 r_{q},\left|y-h_{q}(x)\right|<4 \varepsilon_{q}\right\} \subseteq B_{q} \times J_{q}
$$

holds for some $\varepsilon_{q}, r_{q}>0$. Fix a choice of $R_{q}, B_{q}, J_{q}, h_{q}, r_{q}$, and $\varepsilon_{q}$ for every $q \in \partial \Omega$ and define for all $0<s \leq 4 r_{q}$ and all $0<t \leq 4 \varepsilon_{q}$ :

$$
\begin{aligned}
\tilde{U}_{q}(s, t) & :=R_{q}\left(\left\{(x, y):\left\|x-x_{q}\right\|<s,\left|y-h_{q}(x)\right|<t\right\}\right), \\
\tilde{V}_{q}(s, t) & :=R_{q}\left(\left\{(x, y):\left\|x-x_{q}\right\|<s, h_{q}(x)<y<h_{q}(x)+t\right\}\right)=\tilde{U}_{q}(s, t) \cap \Omega, \\
\tilde{W}_{q}(s, t) & :=R_{q}\left(\left\{(x, y):\left\|x-x_{q}\right\|<s, h_{q}(x) \leq y<h_{q}(x)+t\right\}\right)=\tilde{U}_{q}(s, t) \cap \bar{\Omega} .
\end{aligned}
$$

Then $\left\{\tilde{U}_{q}\left(r_{q}, \varepsilon_{q}\right) \mid q \in \partial \Omega\right\}$ is an open cover of the compact set $\partial \Omega$, and we may find points $q_{i}, i=1, \ldots, n$, such that

$$
\partial \Omega \subseteq \bigcup_{i=1}^{n} \tilde{U}_{q_{i}}\left(r_{q_{i}}, \varepsilon_{q_{i}}\right)
$$

Now consider an arbitrary but fixed set $A \subseteq \bar{\Omega}$ and define

$$
A_{i}:=A \cap \tilde{U}_{q_{i}}\left(r_{q_{i}}, \varepsilon_{q_{i}}\right), \quad i=1, \ldots, n, \quad A_{0}:=A \backslash \bigcup_{i=1}^{n} \tilde{U}_{q_{i}}\left(r_{q_{i}}, \varepsilon_{q_{i}}\right) .
$$

Then

$$
A_{i} \subseteq \tilde{W}_{q_{i}}\left(r_{q_{i}}, \varepsilon_{q_{i}}\right) \quad \forall i=1, \ldots, n, \quad A_{0} \subseteq \Omega \backslash \bigcup_{i=1}^{n} \tilde{U}_{q_{i}}\left(r_{q_{i}}, \varepsilon_{q_{i}}\right), \quad \text { and } \quad A=\bigcup_{i=0}^{n} A_{i},
$$

and we may deduce from Lemma 2.5c) and the (elementary) estimate

$$
\operatorname{cap}_{\Omega}\left(A_{i}\right) \geq \operatorname{cap}_{\tilde{V}_{q_{i}}\left(3 r_{q_{i}}, 3 \varepsilon_{q_{i}}\right)}\left(A_{i}\right) \quad \forall i=1, \ldots, n
$$


that

$$
\begin{aligned}
\operatorname{cap}_{\Omega}(A) & \geq \frac{1}{n+1} \sum_{i=0}^{n} \operatorname{cap}_{\Omega}\left(A_{i}\right) \\
& \geq \frac{1}{n+1}\left(\operatorname{cap}_{\Omega}\left(A_{0}\right)+\sum_{i=1}^{n} \operatorname{cap}_{\tilde{V}_{q_{i}}\left(3 r_{q_{i}}, 3 \varepsilon_{q_{i}}\right)}\left(A_{i}\right)\right) .
\end{aligned}
$$

Note that since

$$
\operatorname{dist}\left(A_{0}, \partial \Omega\right) \geq \operatorname{dist}\left(\Omega \backslash \bigcup_{i=1}^{n} \tilde{U}_{q_{i}}\left(r_{q_{i}}, \varepsilon_{q_{i}}\right), \partial \Omega\right)>0,
$$

Proposition 3.5 and the definitions of $\operatorname{cap}_{\Omega, 0}$ and $\operatorname{cap}_{\Omega^{\prime}, 0}$ imply that there exists a constant $C_{0}>0$ independent of $A$ with

$$
\operatorname{cap}_{\Omega^{\prime}, 0}\left(A_{0}\right) \leq \operatorname{cap}_{\Omega, 0}\left(A_{0}\right) \leq C_{0} \operatorname{cap}_{\Omega}\left(A_{0}\right) .
$$

Accordingly, we just have to estimate the contributions of the sets $A_{i}, i=1, \ldots, n$, in (4.8) to obtain the claim. To this end, fix an $i$, assume w.l.o.g. that $R_{q_{i}}=\mathrm{Id}$, define (as in Lemma 4.2)

$$
U(s, t):=B(s) \times(-t, t), \quad V(s, t):=B(s) \times(0, t), \quad W(s, t):=B(s) \times[0, t),
$$

and let

$$
\Phi_{q_{i}}: U\left(4 r_{q_{i}}, 4 \varepsilon_{q_{i}}\right) \rightarrow \tilde{U}_{q_{i}}\left(4 r_{q_{i}}, 4 \varepsilon_{q_{i}}\right), \quad(x, y) \mapsto\left(x_{q_{i}}+x, y+h_{q_{i}}\left(x_{q_{i}}+x\right)\right) .
$$

Then $\Phi_{q_{i}}$ is bi-Lipschitz with

$$
\Phi_{q_{i}}^{-1}: \tilde{U}_{q_{i}}\left(4 r_{q_{i}}, 4 \varepsilon_{q_{i}}\right) \rightarrow U\left(4 r_{q_{i}}, 4 \varepsilon_{q_{i}}\right), \quad(x, y) \mapsto\left(-x_{q_{i}}+x, y-h_{q_{i}}(x)\right)
$$

and

$$
\begin{aligned}
& \Phi_{q_{i}}\left(\overline{U\left(3 r_{q_{i}}, 3 \varepsilon_{q_{i}}\right)}\right)=\overline{\tilde{U}_{q_{i}}\left(3 r_{q_{i}}, 3 \varepsilon_{q_{i}}\right)}, \\
& \Phi_{q_{i}}\left(\overline{V\left(3 r_{q_{i}}, 3 \varepsilon_{q_{i}}\right)}\right)={\overline{V_{q_{i}}}}_{\left(3 r_{q_{i}}, 3 \varepsilon_{q_{i}}\right)}, \\
& \Phi_{q_{i}}\left(W\left(r_{q_{i}}, \varepsilon_{q_{i}}\right)\right)=\tilde{W}_{q_{i}}\left(r_{q_{i}}, \varepsilon_{q_{i}}\right) \supseteq A_{i} .
\end{aligned}
$$

From the above and Lemma 4.3, we obtain that there exists a $C=C\left(r_{q_{i}}, \varepsilon_{q_{i}}, h_{q_{i}}\right)$ with

$$
\begin{aligned}
& \operatorname{cap}_{V\left(3 r_{q_{i}}, 3 \varepsilon_{q_{i}}\right)}\left(\Phi_{q_{i}}^{-1}\left(A_{i}\right)\right) \leq C \operatorname{cap}_{\tilde{V}_{q_{i}}\left(3 r_{q_{i}}, 3 \varepsilon_{q_{i}}\right)}\left(A_{i}\right) \\
& \operatorname{cap}_{\tilde{U}_{q_{i}}\left(3 r_{q_{i}}, 3 \varepsilon_{q_{i}}\right), 0}\left(A_{i}\right) \leq C \operatorname{cap}_{U\left(3 r_{q_{i}}, 3 \varepsilon_{q_{i}}\right), 0}\left(\Phi_{q_{i}}^{-1}\left(A_{i}\right)\right) .
\end{aligned}
$$

On the other hand, we know that $\Phi_{q_{i}}^{-1}\left(A_{i}\right) \subseteq W\left(r_{q_{i}}, \varepsilon_{q_{i}}\right)$ and, accordingly, we may deduce from Lemma 4.2 that there exists a constant $\tilde{C}=\tilde{C}\left(r_{q_{i}}, \varepsilon_{q_{i}}\right)$ with

$$
\operatorname{cap}_{U\left(3 r_{q_{i}}, 3 \varepsilon_{q_{i}}\right), 0}\left(\Phi_{q_{i}}^{-1}\left(A_{i}\right)\right) \leq \tilde{C} \operatorname{cap}_{V\left(3 r_{q_{i}}, 3 \varepsilon_{q_{i}}\right)}\left(\Phi_{q_{i}}^{-1}\left(A_{i}\right)\right) .
$$

Combining the above, we obtain that there exists a $C_{i}=C_{i}\left(r_{q_{i}}, \varepsilon_{q_{i}}, h_{q_{i}}\right)$ with

$$
\operatorname{cap}_{\Omega^{\prime}, 0}\left(A_{i}\right) \leq \operatorname{cap}_{\tilde{U}_{q_{i}}\left(3 r_{q_{i}}, 3 \varepsilon_{q_{i}}\right), 0}\left(A_{i}\right) \leq C_{i} \operatorname{cap}_{\tilde{V}_{q_{i}}\left(3 r_{q_{i}}, 3 \varepsilon_{q_{i}}\right)}\left(A_{i}\right),
$$


where the first inequality follows from $\tilde{U}_{q_{i}}\left(3 r_{q_{i}}, 3 \varepsilon_{q_{i}}\right) \subseteq \Omega^{\prime}$ and from the definitions of the capacities. We have now proven the existence of positive constants $c_{i}=c_{i}\left(\Omega, \Omega^{\prime}\right)$, $i=0, \ldots, n, c_{i}=1 / C_{i}$, with

$c_{0} \operatorname{cap}_{\Omega^{\prime}, 0}\left(A_{0}\right) \leq \operatorname{cap}_{\Omega}\left(A_{0}\right) \quad$ and $\quad c_{i} \operatorname{cap}_{\Omega^{\prime}, 0}\left(A_{i}\right) \leq \operatorname{cap}_{\tilde{V}_{q_{i}}\left(3 r_{q_{i}}, 3 \varepsilon_{q_{i}}\right)}\left(A_{i}\right), \quad i=1, \ldots, n$.

The above and the subadditivity of the capacity cap $\Omega_{\Omega^{\prime}, 0}$ allow us to continue the estimate in (4.8) as follows

$$
\begin{aligned}
\operatorname{cap}_{\Omega}(A) & \geq \frac{1}{n+1}\left(\operatorname{cap}_{\Omega}\left(A_{0}\right)+\sum_{i=1}^{n} \operatorname{cap}_{\tilde{V}_{q_{i}}\left(3 r_{q_{i}}, 3 \varepsilon_{q_{i}}\right)}\left(A_{i}\right)\right) \\
& \geq \frac{1}{n+1}\left(\sum_{i=0}^{n} c_{i} \operatorname{cap}_{\Omega^{\prime}, 0}\left(A_{i}\right)\right) \\
& \geq \frac{\min \left(c_{0}, \ldots, c_{n}\right)}{n+1} \operatorname{cap}_{\Omega^{\prime}, 0}(A)
\end{aligned}
$$

This proves the claim.

Combining Lemma 4.1 and Proposition 4.4, we obtain that the capacities cap $\Omega$, $\operatorname{cap}_{\Omega^{\prime}, 0}$, and $\operatorname{cap}_{\mathbb{R}^{\mathrm{d}}}$ are indeed equivalent on $\bar{\Omega}$ :

THEOREM 4.5. There exists a constant $C=C\left(\Omega, \Omega^{\prime}\right)$ with

$$
\operatorname{cap}_{\Omega}(A) \leq \operatorname{cap}_{\mathbb{R}^{\mathrm{d}}}(A) \leq \operatorname{cap}_{\Omega^{\prime}, 0}(A) \leq C \operatorname{cap}_{\Omega}(A) \quad \forall A \in \mathcal{P}(\bar{\Omega})
$$

REMARK 4.6. The mere existence of a linear and continuous extension operator $E: W^{1, p}(\Omega) \rightarrow W_{0}^{1, p}\left(\Omega^{\prime}\right)$ is not sufficient for proving (4.9). In order to obtain the reverse estimate in Proposition 4.4, one has to check that there exists such an operator E with

$$
\begin{aligned}
& v \in W^{1, p}(\Omega) \text { and } v \geq 1 \mathcal{L}^{d} \text {-a.e. in } G \in \mathcal{O}(\bar{\Omega}) \\
& \quad \Rightarrow E v \in W_{0}^{1, p}\left(\Omega^{\prime}\right) \text { satisfies } E v \geq 1 \mathcal{L}^{d} \text {-a.e. in some } G^{\prime} \in \mathcal{O}\left(\overline{\Omega^{\prime}}\right) \text { with } G \subseteq G^{\prime} .
\end{aligned}
$$

Note that the extension by reflection employed in the proof of Proposition 4.4 meets this requirement as we have seen in (4.4).

5. The Capacity $\operatorname{cap}_{\partial \Omega}$ on $\mathcal{P}(\partial \Omega)$. In the following section, we show that, in addition to being equivalent to each other on $\bar{\Omega}$, the capacities $\operatorname{cap}_{\Omega}$, $\operatorname{cap}_{\mathbb{R}^{\mathrm{d}}}$, and $\operatorname{cap}_{\Omega^{\prime}, 0}$ are also equivalent to the capacity cap $\partial \Omega$ of the trace space $W^{1-1 / p, p}(\partial \Omega)$ on the boundary $\partial \Omega$. We begin our analysis with the following observation:

Lemma 5.1. For all $A \in \mathcal{P}(\partial \Omega)$,

$$
\operatorname{cap}_{\partial \Omega}(A) \leq \operatorname{cap}_{\Omega}(A)
$$


Proof. From Remark 3.2c) and the definition of $\|\cdot\|_{W^{1-1 / p, p}(\partial \Omega)}$, we gather that for all $A \subseteq \partial \Omega$ :

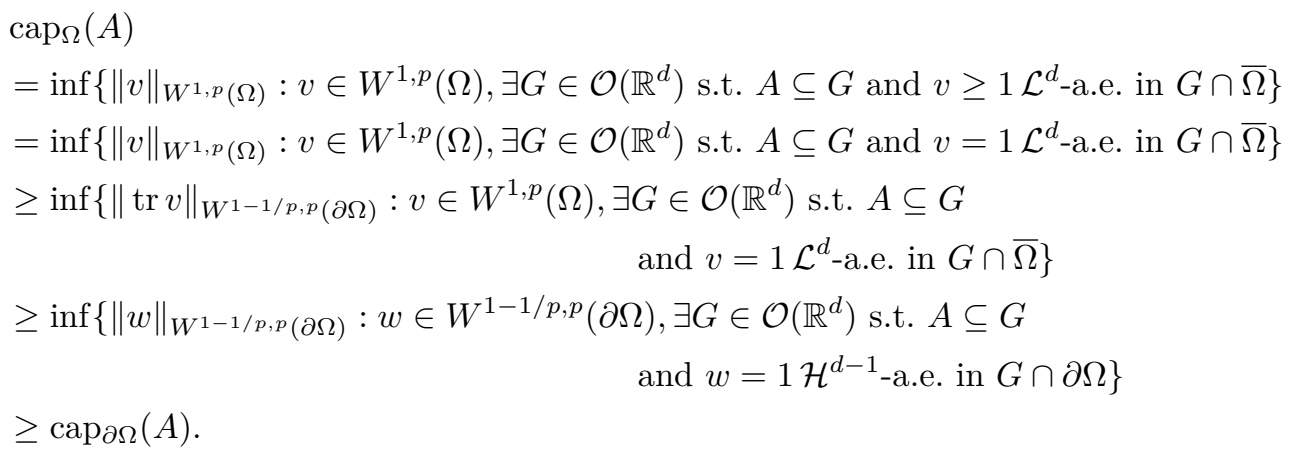

This proves the claim.

To obtain an estimate reverse to (5.1), we use an argumentation similar to the one in Section 4. Recall that the following holds:

Lemma 5.2 ([19, Lemma 5.6]). Let $B(s)$ denote the open ball in $\mathbb{R}^{d-1}$ with radius $s$ centered at the origin, and let $\rho$ be a mollifying kernel on $\mathbb{R}^{d-1}$, i.e.,

$$
0 \leq \rho \in C^{\infty}\left(\mathbb{R}^{d-1}\right), \quad \operatorname{supp}(\rho) \subseteq B(1), \quad \int_{\mathbb{R}^{d-1}} \rho \mathrm{d} \mathcal{L}^{d-1}=1 .
$$

Let $v \in W^{1-1 / p, p}\left(\mathbb{R}^{d-1} \times\{0\}\right) \cong W^{1-1 / p, p}\left(\mathbb{R}^{d-1}\right)$ be a function with $v=0 \mathcal{L}^{d-1}$-a.e. in $\mathbb{R}^{d-1} \backslash B(r), r>0$, and let $R>0$ be arbitrary but fixed. Then there exists a constant $C$ independent of $v$ such that

$$
w\left(x^{\prime}, x_{d}\right):=\frac{1}{x_{d}^{d-1}} \int_{\mathbb{R}^{d-1}} v\left(y^{\prime}\right) \rho\left(\frac{y^{\prime}-x^{\prime}}{x_{d}}\right) \mathrm{d} \mathcal{L}^{d-1}\left(y^{\prime}\right), \quad\left(x^{\prime}, x_{d}\right) \in \mathbb{R}^{d-1} \times(0, R)
$$

satisfies

$$
\begin{gathered}
w \in C^{\infty}\left(\mathbb{R}^{d-1} \times(0, R)\right), \quad \operatorname{tr} w=v \text { on } \mathbb{R}^{d-1} \times\{0\}, \\
w=0 \mathcal{L}^{d} \text {-a.e. in }\left\{\left(x^{\prime}, x_{d}\right) \in \mathbb{R}^{d-1} \times(0, R):\left\|x^{\prime}\right\| \geq r+x_{d}\right\}, \\
\|w\|_{W^{1, p}\left(\mathbb{R}^{d-1} \times(0, R)\right)} \leq C\|v\|_{W^{1-1 / p, p}\left(\mathbb{R}^{d-1} \times\{0\}\right)} .
\end{gathered}
$$

By use of the previous Lemma 5.2, we obtain:

Lemma 5.3. Let $B(s)$ denote the open ball in $\mathbb{R}^{d-1}$ with radius $s$ centered at the origin and let

$$
U(s, t):=B(s) \times(-t, t), \quad V(s, t):=B(s) \times(0, t), \quad R(s):=B(s) \times\{0\} .
$$

Then for all $r, \varepsilon>0$ there exists a constant $C$ independent of $v$ such that

$$
\operatorname{cap}_{U(3 r, 3 \varepsilon), 0}(A) \leq C \operatorname{cap}_{\partial V(3 r, 3 \varepsilon)}(A) \quad \text { for all } A \subseteq R(r) .
$$


Proof. Let $r, \varepsilon>0$ and $A \subseteq R(r)$ be arbitrary but fixed, and suppose that a function $v \in W^{1-1 / p, p}(\partial V(3 r, 3 \varepsilon))$ and a set $G \in \mathcal{O}\left(\mathbb{R}^{d}\right)$ are given such that

$$
A \subseteq G \text { and } v \geq 1 \mathcal{H}^{d-1} \text {-a.e. in } G \cap \partial V(3 r, 3 \varepsilon) .
$$

Let $\psi \in C_{c}^{\infty}\left(\mathbb{R}^{d}\right)$ be an arbitrary but fixed bump function satisfying

$$
\begin{gathered}
\psi \equiv 1 \text { in } U(r, \varepsilon), \quad \psi \in[0,1] \text { in } U(2 r, 2 \varepsilon) \backslash U(r, \varepsilon), \\
\psi \equiv 0 \text { in } \mathbb{R}^{d} \backslash U(2 r, 2 \varepsilon) .
\end{gathered}
$$

Then the function $\psi v$, or rather its extension by zero onto $\mathbb{R}^{d-1} \times\{0\}$, satisfies

$$
\begin{gathered}
\psi v \in W^{1-1 / p, p}\left(\mathbb{R}^{d-1} \times\{0\}\right), \quad \psi v=0 \mathcal{H}^{d-1} \text {-a.e. in }\left(\mathbb{R}^{d-1} \times\{0\}\right) \backslash R(2 r), \\
\psi v=v \geq 1 \mathcal{H}^{d-1} \text {-a.e. in } G \cap R(r), \quad A \subseteq G \cap U(r, \varepsilon),
\end{gathered}
$$

and

$$
\|\psi v\|_{W^{1-1 / p, p}\left(\mathbb{R}^{d-1} \times\{0\}\right)} \leq C\|v\|_{W^{1-1 / p, p}(\partial V(3 r, 3 \varepsilon))}
$$

with a positive constant $C=C(\psi, \varepsilon, r)$. For $\left(x^{\prime}, x_{d}\right) \in \mathbb{R}^{d-1} \times((-3 \varepsilon, 3 \varepsilon) \backslash\{0\})$, we define

$$
w\left(x^{\prime}, x_{d}\right)=\frac{\psi\left(x^{\prime},\left|x_{d}\right|\right)}{\left|x_{d}\right|^{d-1}} \int_{\mathbb{R}^{d-1}}(\psi v)\left(y^{\prime}\right) \rho\left(\frac{y^{\prime}-x^{\prime}}{\left|x_{d}\right|}\right) \mathrm{d} \mathcal{L}^{d-1}\left(y^{\prime}\right),
$$

where $\rho$ denotes a mollifying kernel on $\mathbb{R}^{d-1}$ as in Lemma 5.2. The same lemma and the properties of $\psi$ then imply that $w \in W_{0}^{1, p}(U(3 r, 3 \varepsilon))$ and

$$
\|w\|_{W^{1, p}(U(3 r, 3 \varepsilon))} \leq C\|v\|_{W^{1-1 / p, p}(\partial V(3 r, 3 \varepsilon))}
$$

with a (different) constant $C=C(\psi, \varepsilon, r)$. We claim that $w \geq 1$ holds $\mathcal{L}^{d}$-a.e. in a neighborhood of $A$. To see this, for any $z^{\prime} \in B(r)$ we define

$$
g\left(z^{\prime}\right):=\sup \left\{s>0:\left(z^{\prime}+B(s)\right) \times\{0\} \subseteq G \cap U(r, \varepsilon)\right\} \in\{-\infty\} \cup(0, \infty)
$$

and

$$
H\left(z^{\prime}\right):=\left\{\left(x^{\prime}, x_{d}\right):\left\|x^{\prime}-z^{\prime}\right\|<g\left(z^{\prime}\right)-\left|x_{d}\right|\right\} .
$$

Then $H\left(z^{\prime}\right)$ is open for all $z^{\prime} \in B(r)$, and for all $\left(x^{\prime}, x_{d}\right) \in H\left(z^{\prime}\right)$ we have

$$
\begin{aligned}
\rho\left(\frac{y^{\prime}-x^{\prime}}{\left|x_{d}\right|}\right) \neq 0 & \Rightarrow\left\|\frac{y^{\prime}-x^{\prime}}{x_{d}}\right\| \leq 1 \\
& \Rightarrow\left\|y^{\prime}-z^{\prime}\right\| \leq\left\|y^{\prime}-x^{\prime}\right\|+\left\|x^{\prime}-z^{\prime}\right\|<g\left(z^{\prime}\right) \\
& \Rightarrow\left(y^{\prime}, 0\right) \in G \cap U(r, \varepsilon) .
\end{aligned}
$$

The above yields

$$
w\left(x^{\prime}, x_{d}\right)=\frac{\psi\left(x^{\prime},\left|x_{d}\right|\right)}{\left|x_{d}\right|^{d-1}} \int_{\mathbb{R}^{d-1}}(\psi v)\left(y^{\prime}\right) \rho\left(\frac{y^{\prime}-x^{\prime}}{\left|x_{d}\right|}\right) \mathrm{d} \mathcal{L}^{d-1}\left(y^{\prime}\right) \geq 1
$$

for all $\left(x^{\prime}, x_{d}\right) \in H\left(z^{\prime}\right) \cap U(r, \varepsilon) \backslash\left(\mathbb{R}^{d-1} \times\{0\}\right)$ and thus $w \geq 1 \mathcal{L}^{d}$-a.e. in the open set

$$
H:=U(r, \varepsilon) \cap \bigcup_{z^{\prime} \in B(r)} H\left(z^{\prime}\right) .
$$

Note that $H$ contains $A \subseteq G \cap U(r, \varepsilon) \cap\left(\mathbb{R}^{d-1} \times\{0\}\right)$ according to its definition. 


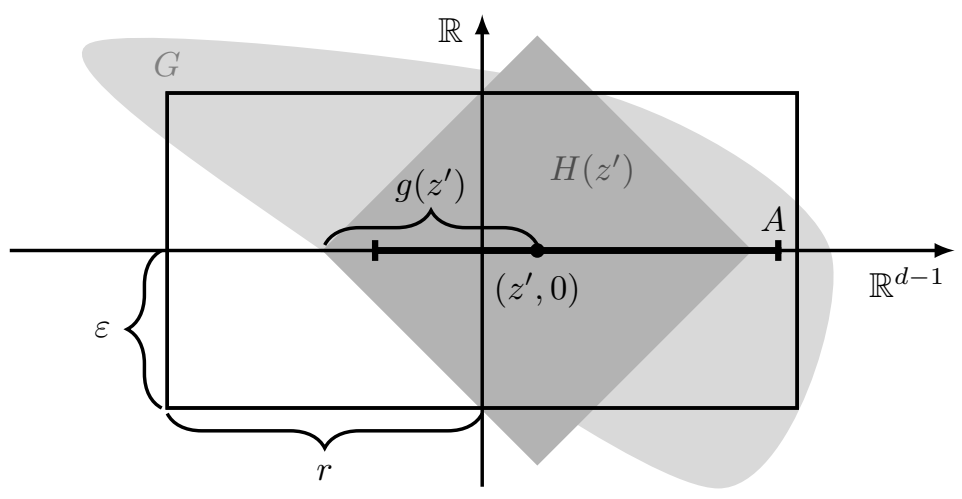

FIG. 5.1. Construction of the set $H\left(z^{\prime}\right)$

As a consequence, the function $w$ satisfies

$$
\begin{gathered}
w \in W_{0}^{1, p}(U(3 r, 3 \varepsilon)), \\
\|w\|_{W^{1, p}(U(3 r, 3 \varepsilon))} \leq C(\psi, \varepsilon, r)\|v\|_{W^{1-1 / p, p}(\partial V(3 r, 3 \varepsilon))}, \\
w \geq 1 \mathcal{L}^{d} \text {-a.e. in } H, \quad H \subseteq U(3 r, 3 \varepsilon) \text { open, } \quad A \subseteq H .
\end{gathered}
$$

Taking the infimum over all $v$ with (5.3) now proves the claim.

To obtain a general result from the prototypical estimate (5.2), we need a stability theorem similar to Lemma 4.3:

LEMMA 5.4. Let $\Omega_{1}, \Omega_{2} \subseteq \mathbb{R}^{d}$ be bounded strong Lipschitz domains, and let $\Omega_{1}^{\prime}, \Omega_{2}^{\prime}$ be open sets satisfying $\overline{\Omega_{i}} \subseteq \Omega_{i}^{\prime}, i=1,2$. Assume that $\Phi: \Omega_{1}^{\prime} \rightarrow \Omega_{2}^{\prime}$ is a bi-Lipschitz mapping with $\Phi\left(\overline{\Omega_{1}}\right)=\overline{\Omega_{2}}$. Then there exist constants $c, C>0$ depending only on $\Phi$ such that

$$
v \circ \Phi \in W^{1-1 / p, p}\left(\partial \Omega_{1}\right) \quad \text { and } \quad\|v \circ \Phi\|_{W^{1-1 / p, p}\left(\partial \Omega_{1}\right)} \leq C\|v\|_{W^{1-1 / p, p}\left(\partial \Omega_{2}\right)}
$$

for every $v \in W^{1-1 / p, p}\left(\partial \Omega_{2}\right)$, and such that

$$
c \operatorname{cap}_{\partial \Omega_{1}}\left(\Phi^{-1}(A)\right) \leq \operatorname{cap}_{\partial \Omega_{2}}(A) \leq C \operatorname{cap}_{\partial \Omega_{1}}\left(\Phi^{-1}(A)\right)
$$

for all $A \subseteq \partial \Omega_{2}$.

Proof. Due to the bi-Lipschitz regularity of $\Phi$, we know $\Phi\left(\partial \Omega_{1}\right)=\partial \Omega_{2}$. Using the identity $\operatorname{tr}(u \circ \Phi)=\operatorname{tr}(u) \circ \Phi$ for all $u \in W^{1, p}\left(\Omega_{2}\right) \cap C\left(\overline{\Omega_{2}}\right)$, the density of $C\left(\overline{\Omega_{2}}\right)$ in $W^{1, p}\left(\Omega_{2}\right)$, and the inverse trace theorem, we readily obtain $v \circ \Phi \in W^{1-1 / p, p}\left(\partial \Omega_{1}\right)$ and

$$
\begin{aligned}
\|v \circ \Phi\|_{W^{1-1 / p, p}\left(\partial \Omega_{1}\right)} & =\inf _{w \in W^{1, p}\left(\Omega_{1}\right): \operatorname{tr} w=v \circ \Phi}\|w\|_{W^{1, p}\left(\Omega_{1}\right)} \\
& \leq \inf _{w \in W^{1, p}\left(\Omega_{2}\right): \operatorname{tr} w=v}\|w \circ \Phi\|_{W^{1, p}\left(\Omega_{1}\right)} \leq C\|v\|_{W^{1-1 / p, p}\left(\partial \Omega_{2}\right)}
\end{aligned}
$$

for all $v \in W^{1-1 / p, p}\left(\partial \Omega_{2}\right)$. Note that the norm estimate (5.5) and the definition of the $W^{1-1 / p, p}$-capacity immediately imply (5.4). This proves the claim.

We combine the previous findings to obtain the following main result of the section.

Proposition 5.5. There exists a constant $C=C\left(\Omega, \Omega^{\prime}\right)>0$ such that

$$
\operatorname{cap}_{\Omega^{\prime}, 0}(A) \leq C \operatorname{cap}_{\partial \Omega}(A) \quad \forall A \subseteq \partial \Omega .
$$


Proof. Let $A \subseteq \partial \Omega$ be arbitrary but fixed, and assume that the quantities $q_{i}, R_{q_{i}}, B_{q_{i}}$ etc. as well as $\tilde{U}_{q_{i}}\left(r_{q_{i}}, \varepsilon_{q_{i}}\right), \tilde{V}_{q_{i}}\left(r_{q_{i}}, \varepsilon_{q_{i}}\right)$ etc. are chosen the same way as in the proof of Proposition 4.4. We define

$$
A_{i}:=A \cap \tilde{U}_{q_{i}}\left(r_{q_{i}}, \varepsilon_{q_{i}}\right), \quad i=1, \ldots, n .
$$

Then $A_{i} \subset \partial \Omega \cap \tilde{U}_{q_{i}}\left(r_{q_{i}}, \varepsilon_{q_{i}}\right)$ for all $i$ and due to Lemma 2.5c)

$$
\operatorname{cap}_{\partial \Omega}(A) \geq \frac{1}{n} \sum_{i=1}^{n} \operatorname{cap}_{\partial \Omega}\left(A_{i}\right) .
$$

Fix an $i$, assume w.l.o.g. that $R_{q_{i}}=\mathrm{Id}$, and choose a cut-off function $\psi_{i} \in C_{c}^{\infty}\left(\mathbb{R}^{d}\right)$ satisfying

$$
\begin{gathered}
\psi_{i} \equiv 1 \text { in } \tilde{U}_{q_{i}}\left(r_{q_{i}}, \varepsilon_{q_{i}}\right), \quad \psi_{i} \in[0,1] \text { in } \tilde{U}_{q_{i}}\left(2 r_{q_{i}}, 2 \varepsilon_{q_{i}}\right) \backslash \tilde{U}_{q_{i}}\left(r_{q_{i}}, \varepsilon_{q_{i}}\right), \\
\psi_{i} \equiv 0 \text { in } \mathbb{R}^{d} \backslash \tilde{U}_{q_{i}}\left(2 r_{q_{i}}, 2 \varepsilon_{q_{i}}\right) .
\end{gathered}
$$

Using Lemma 4.3, Lemma 5.3, Lemma 5.4, $\Phi_{q_{i}}^{-1}\left(A_{i}\right) \subseteq B\left(r_{q_{i}}\right) \times\{0\}$, and the fact that the $W^{1-1 / p, p}$-spaces can equivalently be endowed with the norm (3.2), we obtain the following estimates, where " $\gtrsim$ " denotes greater or equal to up to a positive constant that depends only on $\Omega$ and $\Omega^{\prime}$ :

$$
\begin{aligned}
& \operatorname{cap}_{\partial \Omega}\left(A_{i}\right) \\
& =\inf \left\{\|v\|_{W^{1-1 / p, p}(\partial \Omega)}: v \geq 1 \mathcal{H}^{d-1} \text {-a.e. in a nbhd. of } A_{i}\right\} \\
& \gtrsim \inf \left\{\left\|\psi_{i} v\right\|_{W^{1-1 / p, p}(\partial \Omega)}: v \geq 1 \mathcal{H}^{d-1} \text {-a.e. in a nbhd. of } A_{i}\right\} \\
& \gtrsim \inf \left\{\|v\|_{W^{1-1 / p, p}(\partial \Omega)}: v \geq 1 \mathcal{H}^{d-1} \text {-a.e. in a nbhd. of } A_{i}\right. \text {, } \\
& \left.v=0 \mathcal{H}^{d-1} \text {-a.e. in } \partial \Omega \backslash \tilde{U}_{q_{i}}\left(2 r_{q_{i}}, 2 \varepsilon_{q_{i}}\right)\right\} \\
& \gtrsim \inf \left\{\|v\|_{W^{1-1 / p, p}\left(\partial \tilde{V}\left(3 r_{q_{i}}, 3 \varepsilon_{q_{i}}\right)\right.}: v \geq 1 \mathcal{H}^{d-1} \text {-a.e. in a nbhd. of } A_{i}\right. \text {, } \\
& \left.v=0 \mathcal{H}^{d-1} \text {-a.e. in } \partial \tilde{V}\left(3 r_{q_{i}}, 3 \varepsilon_{q_{i}}\right) \backslash \tilde{U}_{q_{i}}\left(2 r_{q_{i}}, 2 \varepsilon_{q_{i}}\right)\right\} \\
& \gtrsim \operatorname{cap}_{\partial \tilde{V}\left(3 r_{q_{i}}, 3 \varepsilon_{q_{i}}\right)}\left(A_{i}\right) \\
& \gtrsim \operatorname{cap}_{\partial V\left(3 r_{q_{i}}, 3 \varepsilon_{q_{i}}\right)}\left(\Phi_{q_{i}}^{-1}\left(A_{i}\right)\right) \\
& \gtrsim \operatorname{cap}_{U\left(3 r_{q_{i}}, 3 \varepsilon_{q_{i}}\right), 0}\left(\Phi_{q_{i}}^{-1}\left(A_{i}\right)\right) \\
& \gtrsim \operatorname{cap}_{\tilde{U}\left(3 r_{q_{i}}, 3 \varepsilon_{q_{i}}\right), 0}\left(A_{i}\right), \\
& \gtrsim \operatorname{cap}_{\Omega^{\prime}, 0}\left(A_{i}\right) \text {. }
\end{aligned}
$$

Therefore, there exist constants $c_{i}=c_{i}\left(\Omega, \Omega^{\prime}\right)>0$ with $\operatorname{cap}_{\partial \Omega}\left(A_{i}\right) \geq c_{i} \operatorname{cap}_{\Omega^{\prime}, 0}\left(A_{i}\right)$, and, analogously to the proof of Proposition 4.4,

$$
\operatorname{cap}_{\partial \Omega}(A) \geq \frac{1}{n} \sum_{i=1}^{n} \operatorname{cap}_{\partial \Omega}\left(A_{i}\right) \geq \frac{\min \left(c_{1}, \ldots, c_{n}\right)}{n} \operatorname{cap}_{\Omega^{\prime}, 0}(A)
$$

proves the claim.

Note that, since the capacities $\operatorname{cap}_{\Omega}$, $\operatorname{cap}_{\Omega^{\prime}, 0}$, and $\operatorname{cap}_{\mathbb{R}^{\mathrm{d}}}$ are equivalent on $\bar{\Omega}$, Lemma 5.1 and Proposition 5.5 yield that the set functions $\operatorname{cap}_{\Omega}$, $\operatorname{cap}_{\Omega^{\prime}, 0}$, $\operatorname{cap}_{\mathbb{R}^{\mathrm{d}}}$, and $\operatorname{cap}_{\partial \Omega}$ are equivalent on the boundary $\partial \Omega$. This will be stated as a theorem below. 
6. Summary, Conclusions, and Consequences. The results that we have proved in Sections 3 to 5 can be summarized as follows:

Theorem 6.1 (Equivalence of the Various Notions of Sobolev Capacity). Let Assumption 3.1 be satisfied, and let $\operatorname{cap}_{\Omega}$, $\operatorname{cap}_{\Omega, \mathrm{D}}$, $\operatorname{cap}_{\mathbb{R}^{\mathrm{d}}}$, $\operatorname{cap}_{\Omega^{\prime}, 0}$, and cap ${ }$ be defined as in (3.1). Then there exists a constant $C=C\left(\Omega, \Omega^{\prime}\right)$ such that

$$
\operatorname{cap}_{\Omega} \leq \operatorname{cap}_{\mathbb{R}^{\mathrm{d}}} \leq \operatorname{cap}_{\Omega^{\prime}, 0} \leq C \operatorname{cap}_{\Omega} \text { on } \mathcal{P}(\bar{\Omega})
$$

and

$$
\operatorname{cap}_{\partial \Omega} \leq \operatorname{cap}_{\Omega} \leq \operatorname{cap}_{\mathbb{R}^{\mathrm{d}}} \leq \operatorname{cap}_{\Omega^{\prime}, 0} \leq C \operatorname{cap} \partial \Omega \text { on } \mathcal{P}(\partial \Omega)
$$

Moreover,

$$
\operatorname{cap}_{\Omega}(A) \leq \operatorname{cap}_{\Omega, \mathrm{D}}(A) \leq\left(1+\frac{d^{1 / p}}{\operatorname{dist}(A, D)}\right) \operatorname{cap}_{\Omega}(A) \quad \forall A \in \mathcal{P}(\bar{\Omega}),
$$

where

$$
\operatorname{dist}(A, D):=\inf _{x \in A, y \in D}|x-y|
$$

Proof. See Proposition 3.5, Theorem 4.5, Lemma 5.1, and Proposition 5.5.

As a direct consequence of Theorem 6.1, we obtain the following generalization of [21, Lemma A.2] and [16, Corollary 2.39]:

Corollary 6.2 (Equivalence of the Various Notions of Quasi Everywhere). Let Assumption 3.1 be satisfied, and let $\operatorname{cap}_{\Omega}, \operatorname{cap}_{\Omega, \mathrm{D}}$, $\operatorname{cap}_{\mathbb{R}^{\mathrm{d}}}$, $\operatorname{cap}_{\Omega^{\prime}, 0}$, and cap ${ }_{\partial \Omega}$ be defined as in (3.1). Then the following is true for the associated notions of quasi everywhere:

a) If a property holds quasi everywhere in $\bar{\Omega}$ w.r.t. one of the capacities $\operatorname{cap}_{\Omega}$, $\operatorname{cap}_{\Omega^{\prime}, 0}$, and $\operatorname{cap}_{\mathbb{R}^{\mathrm{d}}}$, then it holds quasi everywhere in $\bar{\Omega}$ w.r.t. all of the capacities $\operatorname{cap}_{\Omega}$, $\operatorname{cap}_{\Omega^{\prime}, 0}$, and $\operatorname{cap}_{\mathbb{R}^{\mathrm{d}}}$.

b) If a property holds quasi everywhere on $\partial \Omega$ w.r.t. one of the capacities $\operatorname{cap}_{\Omega}$, $\operatorname{cap}_{\Omega^{\prime}, 0}$, $\operatorname{cap}_{\mathbb{R}^{\mathrm{d}}}$, and $\operatorname{cap}_{\partial \Omega}$, then it holds quasi everywhere on $\partial \Omega$ w.r.t. all of the capacities $\operatorname{cap}_{\Omega}, \operatorname{cap}_{\Omega^{\prime}, 0}$, $\operatorname{cap}_{\mathbb{R}^{\mathrm{d}}}$, and $\operatorname{cap}_{\partial \Omega}$.

c) If a property holds quasi everywhere on $\bar{\Omega} \backslash \bar{D}$ w.r.t. one of the capacities $\operatorname{cap}_{\Omega}$, $\operatorname{cap}_{\Omega^{\prime}, 0}$, $\operatorname{cap}_{\mathbb{R}^{\mathrm{d}}}$, and $\operatorname{cap}_{\Omega, \mathrm{D}}$, then it holds quasi everywhere on $\bar{\Omega} \backslash \bar{D}$ w.r.t. all of the capacities $\operatorname{cap}_{\Omega}$, $\operatorname{cap}_{\Omega^{\prime}, 0}, \operatorname{cap}_{\mathbb{R}^{\mathrm{d}}}$, and $\operatorname{cap}_{\Omega, \mathrm{D}}$.

Proof. Parts $a$ ) and $b$ ) are trivial consequences of Theorem 6.1. To obtain $c$ ), it sufficies to check that every set $A \subseteq \bar{\Omega} \backslash \bar{D}$ with $\operatorname{cap}_{\Omega}(A)=0$ satisfies $\operatorname{cap}_{\Omega, \mathrm{D}}(A)=0$. The remaining implications in $c$ ) follow from part $a$ ) of the corollary and (6.1). So let us consider an arbitrary but fixed set $A \subseteq \bar{\Omega} \backslash \bar{D}$ with $\operatorname{cap}_{\Omega}(A)=0$. Since $\bar{D}$ is a closed set, any element of the complement $\bar{\Omega} \backslash \bar{D}$ has positive distance to $D$, and we may write

$$
A=\bigcup_{n=1}^{\infty}\left\{x \in A: \operatorname{dist}(x, D) \geq \frac{1}{n}\right\}
$$

Proposition 3.5 and the monotonicity of cap $\Omega$ imply that

$$
\operatorname{cap}_{\Omega, \mathrm{D}}\left(\left\{x \in A: \operatorname{dist}(x, D) \geq \frac{1}{n}\right\}\right) \leq\left(1+d^{1 / p} n\right) \operatorname{cap}_{\Omega}(A)=0
$$


for all $n$. The above and the subadditivity of $\operatorname{cap}_{\Omega, \mathrm{D}}($ see Lemma $2.5 d)$ ) yield

$$
\operatorname{cap}_{\Omega, \mathrm{D}}(A) \leq \sum_{n=1}^{\infty} \operatorname{cap}_{\Omega, \mathrm{D}}\left(\left\{x \in A: \operatorname{dist}(x, D) \geq \frac{1}{n}\right\}\right)=0
$$

This proves the claim.

Note that the set $\bar{\Omega} \backslash \bar{D}$ in Corollary $6.2 c$ ) cannot be replaced with $\bar{\Omega} \backslash D$ : If $A$ is a $\operatorname{cap}_{\Omega}$-polar set which intersects $\bar{D}$, then necessarily $\operatorname{cap}_{\Omega, \mathrm{D}}(A)=\infty$, and $A$ is clearly not polar w.r.t. the capacity $\operatorname{cap}_{\Omega, \mathrm{D}}$. For Sobolev functions, we may further conclude: Corollary 6.3. Let Assumption 3.1 be satisfied, let $\operatorname{tr}: W^{1, p}(\Omega) \rightarrow W^{1-1 / p, p}(\partial \Omega)$ be the trace operator, and let $E: W^{1, p}(\Omega) \rightarrow W^{1, p}\left(\mathbb{R}^{d}\right)$ be a continuous extension operator with $E\left(W^{1, p}(\Omega) \cap C(\bar{\Omega})\right) \subseteq C\left(\mathbb{R}^{d}\right)$. Then

$$
v=E v=\operatorname{tr} v \quad \text { q.e. on } \partial \Omega \text { for all } v \in W^{1, p}(\Omega) .
$$

Here, with "q.e. on $\partial \Omega$ " we mean the following: For every cap $_{\Omega}$-quasi continuous representative $\widetilde{v}: \bar{\Omega} \rightarrow \mathbb{R}$ of $v \in W^{1, p}(\Omega)$, every cap $\partial \Omega_{\text {-quasi continuous representative }}$ $(\operatorname{tr} v): \partial \Omega \rightarrow \mathbb{R}$ of the trace $\operatorname{tr} v \in W^{1-1 / p, p}(\partial \Omega)$, and every cap $\mathbb{R}^{\text {d-quasi continuous }}$ representative $(\widetilde{E v}): \mathbb{R}^{d} \rightarrow \mathbb{R}$ of $E v \in W^{1, p}\left(\mathbb{R}^{d}\right)$, there exists a set $N \subseteq \partial \Omega$ such that

$$
\operatorname{cap}_{\Omega}(N)=\operatorname{cap}_{\Omega^{\prime}, 0}(N)=\operatorname{cap}_{\mathbb{R}^{\mathrm{d}}}(N)=\operatorname{cap}_{\partial \Omega}(N)=0
$$

and

$$
\widetilde{v}(x)=\widetilde{(\operatorname{tr} v})(x)=(\widetilde{E v})(x) \quad \forall x \in \partial \Omega \backslash N .
$$

Proof. Let $v_{n} \in W^{1, p}(\Omega) \cap C(\bar{\Omega})$ be a sequence with $v_{n} \rightarrow v$ in $W^{1, p}(\Omega)$. Then we know that

$$
\begin{gathered}
C(\partial \Omega) \cap W^{1-1 / p, p}(\partial \Omega) \ni \operatorname{tr} v_{n} \rightarrow \operatorname{tr} v \in W^{1-1 / p, p}(\partial \Omega), \\
C\left(\mathbb{R}^{d}\right) \cap W^{1, p}\left(\mathbb{R}^{d}\right) \ni E v_{n} \rightarrow E v \in W^{1, p}\left(\mathbb{R}^{d}\right) .
\end{gathered}
$$

Corollary 2.11 implies the existence of a subsequence, which we still denote by $v_{n}$, such that

$$
\begin{aligned}
v_{n}(x) & \rightarrow \tilde{v}(x) \quad \forall x \in \bar{\Omega} \backslash N_{1}, \\
\left(\operatorname{tr} v_{n}\right)(x) & \rightarrow(\widetilde{\operatorname{tr} v})(x) \quad \forall x \in \partial \Omega \backslash N_{2}, \\
\left(E v_{n}\right)(x) & \rightarrow(\widetilde{E v})(x) \quad \forall x \in \mathbb{R}^{d} \backslash N_{3},
\end{aligned}
$$

where $\tilde{v},(\widetilde{\operatorname{tr} v})$, and $(\widetilde{E v})$ are quasi continuous representatives of $v, \operatorname{tr} v$, and $E v$, respectively, and where $N_{1} \subseteq \bar{\Omega}, N_{2} \subseteq \partial \Omega$, and $N_{3} \subseteq \mathbb{R}^{d}$ satisfy

$$
\operatorname{cap}_{\Omega}\left(N_{1}\right)=\operatorname{cap}_{\partial \Omega}\left(N_{2}\right)=\operatorname{cap}_{\mathbb{R}^{\mathrm{d}}}\left(N_{3}\right)=0 .
$$

Defining $N=\left(N_{1} \cup N_{2} \cup N_{3}\right) \cap \partial \Omega$, the claim now follows from $v_{n}=\operatorname{tr} v_{n}=E v_{n}$ on $\partial \Omega$ for all $n$, the uniqueness of quasi continuous representatives up to polar sets (cf. Theorem 2.13), and the equivalencies in Theorem 6.1.

REMARK 6.4. Corollary 6.3 yields that the restriction of a cap $\Omega^{-q u a s i}$ continuous representative to the boundary $\partial \Omega$ is always a cap $\Omega^{-}$-quasi continuous representative 
of the trace. This result seems to not have been proven so far in the literature but accords very well with the intuition, cf. [26, Rem. 4.4.5, Ex. 4.2] for an approach to the trace operator by extensions of Sobolev functions that are unique w.r.t. $\mathcal{H}^{d-1}$.

ReMARK 6.5. We emphasize once more that the results in Theorem 6.1, Corollary 6.2, and Corollary 6.3 are easily transformed to conform to the definitions of Sobolev capacity employed in [5, 8, 11, 16] etc. To do so, one simply has to raise the left- and the right-hand sides of the capacity estimates to the power $p$.

We conclude our analysis with an application of Corollary 6.3 that is relevant for the study of Signorini-type variational inequalities:

Corollary 6.6. Consider the situation in Assumption 3.1, let $\psi: \partial \Omega \rightarrow \mathbb{R} \cup\{ \pm \infty\}$ be a given function, and let

$$
\widehat{\psi}(x):=\left\{\begin{array}{ll}
\psi(x) & \text { if } x \in \partial \Omega \\
-\infty & \text { if } x \in \Omega
\end{array},\right.
$$

then

$$
\begin{aligned}
\{v & \left.\in W^{1, p}(\Omega): v \geq \widehat{\psi} \operatorname{cap}_{\Omega} \text {-q.e. on } \bar{\Omega}\right\} \\
& =\left\{v \in W^{1, p}(\Omega): v \geq \widehat{\psi} \operatorname{cap}_{\Omega^{\prime}, 0}-q . e . \text { on } \bar{\Omega}\right\} \\
& =\left\{v \in W^{1, p}(\Omega): v \geq \widehat{\psi} \operatorname{cap}_{\mathbb{R}^{\mathrm{d}}} \text {-q.e. on } \bar{\Omega}\right\} \\
& =\left\{v \in W^{1, p}(\Omega): \operatorname{tr} v \geq \psi \operatorname{cap} \partial \Omega \text {-q.e. on } \partial \Omega\right\} .
\end{aligned}
$$

If, additionally, $\psi$ is cap $\partial \Omega$-quasi lower semi-continuous on $\partial \Omega$, then we also have

$$
\begin{aligned}
& \left\{v \in W^{1, p}(\Omega): \operatorname{tr} v \geq \psi \text { cap } \partial \Omega \text {-q.e. on } \partial \Omega\right\} \\
& \quad=\left\{v \in W^{1, p}(\Omega): \operatorname{tr} v \geq \psi \mathcal{H}^{d-1} \text {-a.e. on } \partial \Omega\right\} .
\end{aligned}
$$

Proof. The identities in (6.2) are trivial consequences of Corollary 6.2 and Corollary 6.3. The equality in (6.3) follows from Lemma 2.5a) and Lemma 2.12.

The above result shows that the approaches to the differential sensitivity analysis of Signorini-type problems employed in [17, Exemple 2, page 150], [15, Example 6], and [6, Section 3.2] are exactly the same. The notions of capacity used by the authors of the latter papers are equivalent and so are the results that they arrive at. Note

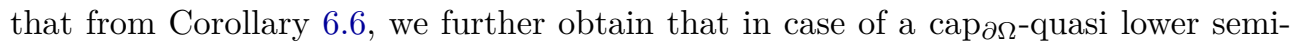
continuous obstacle $\psi$, we may replace the inequality $\operatorname{tr} v \geq \psi \mathcal{H}^{d-1}$-a.e. on $\partial \Omega$ in (6.3) by either of the inequalities in (6.2) without changing the described subset of $W^{1, p}(\Omega)$. This shows that, under very weak assumptions on the obstacle $\psi$ and the domain $\Omega$, the $\mathcal{H}^{d-1}$-description of the admissible set of a Signorini-type problem, which is often used in numerics, see [9, 13], is identical to the capacity description, which is natural in the sensitivity analysis. We point out that it is not possible to obtain, e.g., the identity

$$
\begin{aligned}
& \left\{v \in W^{1, p}(\Omega): v \geq \widehat{\psi} \operatorname{cap}_{\Omega} \text {-q.e. on } \bar{\Omega}\right\} \\
& \quad=\left\{v \in W^{1, p}(\Omega): \operatorname{tr} v \geq \psi \mathcal{H}^{d-1} \text {-a.e. on } \partial \Omega\right\}, \quad \psi \operatorname{cap}_{\partial \Omega} \text {-q.l.s.c., }
\end{aligned}
$$

directly from the analysis in Section 2 (and in particular not from Lemma 2.12). Since the function $\widehat{\psi}$ typically has no lower semi-continuity properties whatsoever, to obtain (6.4), one necessarily has to take the detour via the boundary capacity capər. 


\section{References.}

[1] D.R. Adams. Choquet integrals in potential theory. Publicacions Matemàtiques, 42(1):3-66, 1998.

[2] D.R. Adams and L.I. Hedberg. Function Spaces and Potential Theory. Springer Verlag, Berlin, 1999.

[3] R.A. Adams and J.J.F. Fournier. Sobolev Spaces. Elsevier, Amsterdam, second edition, 2003.

[4] L. Ambrosio, G. Da Prato, and A. Mennucci. Introduction to Measure Theory and Integration. Edizioni Della Normale, Pisa, 2011.

[5] H. Attouch, G. Buttazzo, and G. Michaille. Variational Analysis in Sobolev and BV Spaces: Applications to PDEs and Optimization. SIAM, Philadelphia, PA, 2006.

[6] T. Betz. Optimal control of two variational inequalities arising in solid mechanics. $\mathrm{PhD}$ thesis, Technische Universität Dortmund, 2015.

[7] A. Beurling and J. Deny. Dirichlet spaces. Proc. Natl. Acad. Sci. USA, 45(2): 208-215, 1959.

[8] J.F. Bonnans and A. Shapiro. Perturbation Analysis of Optimization Problems. Springer Verlag, New York, 2000.

[9] P.G. Ciarlet. Lectures on the Finite Element Method. Tata Institute of Fundamental Research, Bombay, 1975.

[10] M. Egert, R. Haller-Dintelmann, and J. Rehberg. Hardy's inequality for functions vanishing on a part of the boundary. Potential Anal., 43(1):49-78, 2015.

[11] L.C. Evans and R.F. Gariepy. Measure theory and fine properties of functions. CRC Press, Boca Raton, FL, revised edition, 2015.

[12] M. Fukushima. Dirichlet forms and Markov Processes. North-Holland, Amsterdam, 1980.

[13] R. Glowinski. Lectures on Numerical Methods for Non-Linear Variational Problems. Tata Institute of Fundamental Research, Bombay, 1980.

[14] P. Grisvard. Elliptic problems in nonsmooth domains. Pitman, Boston, MA, 1985.

[15] A. Haraux. How to differentiate the projection on a convex set in Hilbert space. Some applications to variational inequalities. J. Math. Soc. Japan, 29(4):615-631, 1977.

[16] J. Heinonen, T. Kilpeläinen, and O. Martio. Nonlinear Potential Theory of Degenerate Elliptic Equations. Dover Publications, New York, 2006.

[17] F. Mignot. Contrôle dans les inéquations variationelles elliptiques. J. Funct. Anal., 22(2):130-185, 1976.

[18] G. Müller and A. Schiela. On the control of time discretized dynamical contact problems. Submitted, 2015.

[19] J. Nečas. Direct Methods in the Theory of Elliptic Equations. Springer Verlag, Berlin, 2012.

[20] Jaak Peetre. A counterexample connected with Gagliardo's trace theorem. Comment. Math. Special Issue, 2:277-282, 1979. Special issue dedicated to Władysław Orlicz on the occasion of his seventy-fifth birthday.

[21] G. Wachsmuth. Strong stationarity for optimal control of the obstacle problem with control constraints. SIAM J. Optim., 24(4):1914-1932, 2014.

[22] G. Wachsmuth. Towards M-stationarity for optimal control of the obstacle problem with control constraints. SIAM J. Control Optim., 54(2):964-986, 2016.

[23] G. Wachsmuth. Pointwise constraints in vector-valued Sobolev spaces. Appl. 
Math. Optim., pages 1-35, 2016.

[24] A. Wilanski. Topology for Analysis. Ginn, Waltham, MA, 1970.

[25] S. Willard. General Topology. Addison-Wesley, London, 1970.

[26] W.P. Ziemer. Weakly differentiable functions. Springer Verlag, New York, 1989. 